\title{
EXTENSION BY ZERO IN DISCRETE TRACE SPACES: INVERSE ESTIMATES
}

\author{
RALF HIPTMAIR, CARLOS JEREZ-HANCKES, AND SHIPENG MAO
}

\begin{abstract}
We consider lowest-order $\boldsymbol{H}^{-\frac{1}{2}}\left(\operatorname{div}_{\Gamma}, \Gamma\right)$ - and $H^{-\frac{1}{2}}(\Gamma)$-conforming boundary element spaces supported on part of the boundary $\Gamma$ of a Lipschitz polyhedron. Assuming families of triangular meshes created by regular refinement, we prove that on these spaces the norms of the extension by zero operators with respect to (localized) trace norms increase poly-logarithmically with the mesh width. Our approach harnesses multilevel norm equivalences for boundary element spaces, inherited from stable multilevel splittings of finite element spaces.
\end{abstract}

\section{INTRODUCTION}

We consider a bounded Lipschitz polyhedron $\Omega \subset \mathbb{R}^{3}$ with trivial topology and set $\Gamma:=\partial \Omega$ to be its compact boundary composed of a small number of flat faces. Write $\Gamma^{+} \subsetneq \Gamma$ for an open part of the boundary of $\Omega$. We assume that it is simply connected and agrees with the interior of a union of some closed faces of $\Omega$.

Given a function $u: \Gamma^{+} \rightarrow \mathbb{R}$ we can consider its extension by zero, $\mathrm{Z} u: \Gamma \rightarrow \mathbb{R}$, defined for continuous $u$ by

$$
\mathrm{Z} u(\boldsymbol{x})= \begin{cases}u(\boldsymbol{x}), & \text { for } \boldsymbol{x} \in \Gamma^{+}, \\ 0, & \text { for } \boldsymbol{x} \in \Gamma \backslash \Gamma^{+} .\end{cases}
$$

For a Sobolev space $X$ of generalized functions on $\Gamma$, the domain of this operator is the subspace

$$
\widetilde{X}:=\{u \in X, \mathrm{Z} u \in X\} .
$$

The situation is crystal clear for Sobolev spaces $L_{2}$ and $H^{1}$ : the mapping $\mathbf{Z}$ : $L_{2}\left(\Gamma^{+}\right) \rightarrow L_{2}(\Gamma)$ is trivially continuous, whereas Z : $H^{1}\left(\Gamma^{+}\right) \rightarrow H^{1}(\Gamma)$ is not bounded. This impasse is remedied by restricting $Z$ to the closed subspace $H_{0}^{1}\left(\Gamma^{+}\right)$, which renders Z : $H_{0}^{1}\left(\Gamma^{+}\right) \rightarrow H^{1}(\Gamma)$ an isometry, from which we conclude the isometric isomorphism $\widetilde{H}^{1}(\Gamma) \cong H_{0}^{1}\left(\Gamma^{+}\right)$.

Simplicity ends when considering the fractional Sobolev spaces $H^{\frac{1}{2}}(\Gamma)$ and $H^{\frac{1}{2}}\left(\Gamma^{+}\right)$, which arise from interpolating between $H^{1}$ and $L_{2}$, e.g., $H^{\frac{1}{2}}\left(\Gamma^{+}\right)=$

Received by the editor October 22, 2012 and, in revised form, March 18, 2014.

2010 Mathematics Subject Classification. Primary 65N12, 65N15, 65N30.

Key words and phrases. Boundary finite element spaces, inverse estimates, multilevel norm equivalences.

The work of the second author was funded by FONDECYT 11121166 and CONICYT project Anillo ACT1118 (ANANUM).

The work of the third author was partly supported by Thales SA under contract "Preconditioned Boundary Element Methods for Electromagnetic Scattering at Dielectric Objects" and NSFC 11101414, 11101386, 11471329. 
$\left[L_{2}\left(\Gamma^{+}\right), H^{1}\left(\Gamma^{+}\right)\right]_{\frac{1}{2}}$ 35, Ch. 1, 9.1]. These spaces are very important as (localized) trace spaces of $H^{1}(\Omega)$; see, for example [44, Ch. 13] or [35, Sect. 1.8]. As in the case of $H^{1}$, the operator Z turns out to be unbounded as a mapping $H^{\frac{1}{2}}\left(\Gamma^{+}\right) \mapsto H^{\frac{1}{2}}(\Gamma)$, [35, Thm. 11.4]. Even more striking, according to [35, Thm. 11.7]:

The spact $1 \widetilde{H}^{\frac{1}{2}}\left(\Gamma^{+}\right)$is a dense subspace of $H^{\frac{1}{2}}\left(\Gamma^{+}\right)$, whose graph norm is strictly stronger than the norm of $H^{\frac{1}{2}}\left(\Gamma^{+}\right)$!

This last fact must be reflected by the so-called inverse inequalities connecting the $H^{\frac{1}{2}}\left(\Gamma^{+}\right)$- and $\widetilde{H}^{\frac{1}{2}}\left(\Gamma^{+}\right)$-norms of piecewise polynomial functions in $H^{\frac{1}{2}}(\Gamma)$. Concretely, let us imagine that $\Gamma$ is equipped with a conforming triangular mesh $\Gamma_{h}$ as described in [42, Sect. 4.1.2]. The mesh $\Gamma_{h}$ is supposed to resolve the faces of $\Omega$ in the sense that each face is triangulated by the restriction of $\Gamma_{h}$ to it. In particular, $\Gamma_{h}^{+}:=\left.\Gamma_{h}\right|_{\Gamma^{+}}$provides a mesh of $\Gamma^{+}$. On $\Gamma_{h}$ we consider the finite element space $\mathcal{S}_{1}\left(\Gamma_{h}\right) \subset H^{1}(\Gamma)$ of $\Gamma_{h}$-piecewise linear, globally continuous functions on $\Gamma$; see [42, Sect. 4.1.7]. Their restrictions to $\Gamma^{+}$form the space $\mathcal{S}_{1}\left(\Gamma_{h}^{+}\right)$. We write $\widetilde{\mathcal{S}}_{1}\left(\Gamma_{h}^{+}\right)$for the subspace of functions in $\mathcal{S}_{1}\left(\Gamma_{h}^{+}\right)$that vanish on $\partial \Gamma^{+}$. Since $\widetilde{\mathcal{S}}_{1}\left(\Gamma_{h}^{+}\right) \subset H_{0}^{1}\left(\Gamma^{+}\right)$, we certainly have $\widetilde{\mathcal{S}}_{1}\left(\Gamma_{h}^{+}\right) \subset \widetilde{H}^{\frac{1}{2}}\left(\Gamma^{+}\right)$.

Of course, the $H^{\frac{1}{2}}\left(\Gamma^{+}\right)$- and $\widetilde{H}^{\frac{1}{2}}\left(\Gamma^{+}\right)$-norms are equivalent on the finite dimensional space $\widetilde{\mathcal{S}}_{1}\left(\Gamma_{h}^{+}\right)$. However, owing to the fact highlighted above, their ratio must blow up on very fine meshes $\Gamma_{h}^{+}$, because of "asymptotic density" of $\widetilde{\mathcal{S}}_{1}\left(\Gamma^{+}\right)$ in $\widetilde{H}^{\frac{1}{2}}\left(\Gamma^{+}\right)$as the mesh width $h$ of $\Gamma_{h}^{+}$tends to zero. Quantifying this blow-up as a function of $h$ leads to inverse inequalities, which constitute key elements

- in the theory of non-overlapping domain decomposition preconditioners for second-order elliptic boundary value problems known as sub-structuring methods [45, Ch. 4] and [47, Sect. 5];

- for gauging the effect of operator preconditioning of discrete boundary integral operators for screen problems or multi-domain transmission problems; see [28, 37.

Small wonder, much effort has been devoted to obtaining sharp inverse estimates of the form

$$
\left\|\widetilde{u}_{h}\right\|_{\widetilde{H}^{\frac{1}{2}\left(\Gamma^{+}\right)}} \leq C b(h)\left\|\widetilde{u}_{h}\right\|_{H^{\frac{1}{2}}\left(\Gamma^{+}\right)}, \quad \forall \widetilde{u}_{h} \in \widetilde{\mathcal{S}}_{1}\left(\Gamma_{h}^{+}\right)
$$

where $C>0$ may depend on shape-regularity and quasi-uniformity of $\Gamma_{h}^{+}$, but not on its mesh width. The goal is to characterize the possible functions $b: \mathbb{R}^{+} \rightarrow \mathbb{R}^{+}$ as precisely as possible. This has been achieved in the so-called face lemmas in the theory of sub-structuring domain decomposition methods; see [45, Lemma 4.26] and [47. Lemma 4.10]. They assert that logarithmic bounds of the form $b(h)=1+|\log h|$ hold. The occurrence of logarithms in $b(h)$ is not surprising, since the lack of continuity of extension by zero for $H^{\frac{1}{2}}\left(\Gamma^{+}\right)$is a borderline case in the Sobolev scale $H^{s}\left(\Gamma^{+}\right), 0 \leq s \leq 1$ : for any $s \neq \frac{1}{2}$ the norms of $\widetilde{H}^{s}\left(\Gamma^{+}\right)$and $H^{s}\left(\Gamma^{+}\right)$are equivalent [35. Thm. 11.4]. Inverse inequalities with logarithmic blow-up in terms of the mesh width are typical for such borderline cases and piecewise polynomial finite element spaces of fixed degree [20, Lemma 1.142].

Thus, the case for $H^{\frac{1}{2}}(\Gamma)$ and Lagrangian boundary element spaces is settled, but similar inverse inequalities have remained unexplored for the trace spaces

\footnotetext{
${ }^{1}$ In 35 and other works the space $\widetilde{H}^{\frac{1}{2}}\left(\Gamma^{+}\right)$is denoted by $H_{00}^{\frac{1}{2}}\left(\Gamma^{+}\right)$.
} 
$\boldsymbol{H}^{-\frac{1}{2}}\left(\operatorname{div}_{\Gamma}, \Gamma\right)$ and $H^{-\frac{1}{2}}(\Gamma)$ of $\boldsymbol{H}(\operatorname{curl}, \Omega)$ and $\boldsymbol{H}(\operatorname{div}, \Omega)$, respectively, as well as for associated piecewise polynomial boundary element spaces. Inverse inequalities in these settings are important for the very same reasons that account for interest in (1.3): they are tools for the numerical analysis of domain decomposition methods for $\boldsymbol{H}(\mathbf{c u r l}, \Omega)$-elliptic boundary value problems [32,33], and they are essential for the analysis of operator preconditioning in a boundary element context.

In this article we prove inverse inequalities analogous to (1.3) for low-order $\boldsymbol{H}^{-\frac{1}{2}}\left(\operatorname{div}_{\Gamma}, \Gamma\right)$ - and $H^{-\frac{1}{2}}(\Gamma)$-conforming boundary element spaces. Similarly, as for $H^{\frac{1}{2}}(\Gamma)$, we achieve (poly-)logarithmic bounds for sequences of meshes created by successive regular refinements. Our new idea is the use of multilevel norm equivalences for boundary element spaces borrowed from the theory of multilevel preconditioners [29]. They facilitate control over the impact of extension-by-zero by distributing its effects over all levels in the multilevel hierarchy of spaces. In a sense, this article can be viewed as a supplement to [29] demonstrating another use of the multilevel estimates obtained there.

In parts, developments will rely on ideas introduced in 29. In particular, this applies to the discrete extension operators analyzed in Section 4 and the reasoning in Section 5. Section 3 is special, since it addresses known inverse inequalities (1.3). We have included this section in order to convey the gist of the multilevel approach and the reader is advised to study Section 3 before turning to the more technical subsequent sections. Readers interested in the estimates alone will find our main results at the end of Section 2 in Theorems 2.1 and 2.2, whose proofs are postponed to Section 6 .

Remark 1.1. The considerations presented in this article can be adapted to curvilinear polyhedra with general topology. This does not require essentially new ideas, but would complicate the presentation on the level of technical details. Thus, we have decided to forgo generality for the sake of clarity of presentation. The same applies to the use of boundary element spaces of (fixed) higher polynomial degree.

Remark 1.2. We came to the conclusion that no complete proof of the "face lemma" in the theory of sub-structuring domain decomposition methods is available in the literature. Indeed, all proofs of [19, Lemma 3], [9, Lemma 4.2], and [47, Lemma 4.8] rely on a spurious application of an inverse inequality linking $H^{1}(\Omega)$-norms and $L_{\infty}(\Omega)$-norms of piecewise linear continuous functions; the families of (2D slice) meshes on which these functions are defined lack the required shape-regularity and quasi-uniformity.

\section{TRACES AND BOUNDARY ELEMENT SPACES}

In order to keep the article reasonably self-contained, in this section we recall traces and various low-order boundary element spaces. In passing, we introduce key notations closely following [29, Sect. 3]. For smooth functions and vector fields on $\bar{\Omega}$ we consider the

$$
\begin{array}{ll}
\text { pointwise trace } & \mathrm{T}_{\boldsymbol{x}} v(\boldsymbol{x}):=v(\boldsymbol{x}), \quad \boldsymbol{x} \in \Gamma, \quad \forall v \in C^{\infty}(\bar{\Omega}), \\
\text { tangential trace } & \mathrm{T}_{\boldsymbol{t}} \mathbf{v}(\boldsymbol{x}):=\mathbf{v}(\boldsymbol{x}) \times \boldsymbol{n}(\boldsymbol{x}), \quad \boldsymbol{x} \in \Gamma, \quad \forall \mathbf{v} \in\left(C^{\infty}(\bar{\Omega})\right)^{3}, \\
\text { normal trace } & \mathrm{T}_{\boldsymbol{n}} \mathbf{v}(\boldsymbol{x}):=\mathbf{v}(\boldsymbol{x}) \cdot \boldsymbol{n}(\boldsymbol{x}), \quad \boldsymbol{x} \in \Gamma, \quad \forall \mathbf{v} \in\left(C^{\infty}(\bar{\Omega})\right)^{3} .
\end{array}
$$

These mappings can be extended to continuous and surjective trace operators $\mathrm{T}_{\boldsymbol{x}}: H^{1}(\Omega) \longrightarrow H^{\frac{1}{2}}(\Gamma)$ [36, Thm. 3.3.7], $\mathrm{T}_{\boldsymbol{t}}: \boldsymbol{H}(\mathbf{c u r l}, \Omega) \longrightarrow \boldsymbol{H}^{-\frac{1}{2}}\left(\operatorname{div}_{\Gamma}, \Gamma\right)$ 
[13. Thm. 4.1], and $\mathrm{T}_{\boldsymbol{n}}: \boldsymbol{H}(\operatorname{div}, \Omega) \longrightarrow H^{-\frac{1}{2}}(\Gamma)$ [21, Thm. 2.5]. Here we adopt the notations of [11] and recall that the norm of $\boldsymbol{H}^{-\frac{1}{2}}\left(\operatorname{div}_{\Gamma}, \Gamma\right)$ is defined as

$$
\|\boldsymbol{\psi}\|_{\boldsymbol{H}^{-\frac{1}{2}}\left(\operatorname{div}_{\Gamma}, \Gamma\right)}^{2}:=\|\boldsymbol{\psi}\|_{\boldsymbol{H}_{\|}^{-\frac{1}{2}}(\Gamma)}^{2}+\left\|\operatorname{div}_{\Gamma} \boldsymbol{\psi}\right\|_{H^{-\frac{1}{2}}(\Gamma)}^{2}, \quad \boldsymbol{\psi} \in \boldsymbol{H}^{-\frac{1}{2}}\left(\operatorname{div}_{\Gamma}, \Gamma\right)
$$

where $\boldsymbol{H}_{\|}^{-\frac{1}{2}}(\Gamma)$ is the $L_{2}(\Gamma)$-dual of $\boldsymbol{H}_{\|}^{\frac{1}{2}}(\Gamma)$, the tangential trace space of $\boldsymbol{H}^{1}(\Omega)$.

Notation. We try to distinguish functions from different spaces by font and typeface. Small regular roman letters will designate functions in $H^{\frac{1}{2}}(\Gamma)$ and the corresponding boundary elements spaces. For tangential surface vector fields in $\boldsymbol{H}^{-\frac{1}{2}}\left(\operatorname{div}_{\Gamma}, \Gamma\right)$ we use roman font but bold typeface, whereas elements of $H^{-\frac{1}{2}}(\Gamma)$ are represented by small Greek letters in normal print. Capital roman characters denote functions on $\Omega$, with bold typeface reserved for vector fields. All functions in discrete spaces will bear an integer subscript indicating the refinement level of the mesh on which they are defined.

As in the Introduction, we fix a triangular surface mesh $\Gamma_{0}$ that resolves the faces of $\Omega$. We make the assumption that there is a tetrahedral mesh $\Omega_{0}$ covering $\Omega$ such that $\left.\Omega_{0}\right|_{\Gamma}=\Gamma_{0}$. For a Lipschitz polyhedron this assumption is not particularly restrictive. Starting from $\Omega_{0}$ we create a sequence of nested tetrahedral meshes $\Omega_{0} \prec \Omega_{1} \prec \ldots \prec \Omega_{l} \prec \ldots$ by global regular refinement as described in [6] or [34. Their restrictions to $\Gamma$ spawn a sequence of nested surface meshes $\left(\Gamma_{l}\right)_{l=0}^{\infty}$, $\Gamma_{l}:=\left.\Omega_{l}\right|_{\Gamma}$. In the sequel, we are going to write $\mathcal{V}\left(\Omega_{l}\right) / \mathcal{V}\left(\Gamma_{l}\right), \mathcal{E}\left(\Omega_{l}\right) / \mathcal{E}\left(\Gamma_{l}\right), \mathcal{F}\left(\Omega_{l}\right)$ for sets of vertices, edges, and faces of the meshes $\Omega_{l}$ and $\Gamma_{l}$, respectively.

Appealing to the findings of Bey [6] and Kossaczký [34, we point out that global regular refinement leads to shape-regular families of meshes [42, Def. 4.1.2], that is,

$$
\exists C_{s}>0: \quad \max _{T \in \Gamma_{l}} \frac{h_{T}}{\rho_{T}}, \max _{K \in \Omega_{l}} \frac{h_{K}}{\rho_{K}} \leq C_{s}, \quad \forall l \in \mathbb{N}_{0} .
$$

Here we adopted the conventional notations $h_{T}, h_{K}$ for the diameter of a mesh cell (element), and $\rho_{T}, \rho_{K}$ for the radii of the largest inscribed circle or ball. We set $h_{l}=\max _{T \in \Gamma_{l}} h_{T}$ for the mesh width on level $l$. Regular refinement also ensures quasi-uniformity [42, Def. 4.1.113] in the sense that

$$
\exists C_{u}>0: \quad C_{u}^{-1} \leq \frac{\max _{T \in \Gamma_{l}} h_{T}}{\min _{T \in \Gamma_{l}} h_{T}}, \frac{\max _{K \in \Omega_{l}} h_{K}}{\min _{K \in \Omega_{l}} h_{K}} \leq C_{u}, \quad \forall l \in \mathbb{N}_{0} .
$$

The constants $C_{s}$ and $C_{u}$ depend only on $\Omega_{0}$. Regular refinement makes the mesh width $h_{l}$ satisfy $h_{l} \approx 2^{-l} h_{0}$.

Notation. Following [46], we often write $\approx, \lesssim$, and $\gtrsim$ for two-sided and one-sided inequalities involving constants that may depend only on $\Omega_{0}$ and $\Gamma^{+}$, and, thus, indirectly, on $\Omega, C_{s}, C_{u}$ from (2.2) and (2.3).

After the acceptance of this manuscript the authors learned about the work 1 where discrete extension results in the spirit of Lemma 4.4 and Lemma 4.5] are shown without assuming quasi-unformity of the family of meshes; see 1, Theorems 2.1 and 2.3]. We believe that using the techniques of [1] it will be possible to extend the results of this article to shape-regular families of meshes that fail to satisfy (2.3).

Let $\mathcal{S}_{1}\left(\Omega_{l}\right), \mathcal{N} \mathcal{D}_{1}\left(\Omega_{l}\right)$, and $\mathcal{R} \mathcal{T}_{0}\left(\Omega_{l}\right)$ designate the finite element spaces of $H^{1}(\Omega)$-conforming, piecewise linear (scalar) Lagrangian finite elements, of 
$\boldsymbol{H}(\mathbf{c u r l}, \Omega)$-conforming lowest-order Nédélec elements (edge elements, Whitney 1forms, 26, 38), and, $\boldsymbol{H}$ (div, $\Omega$ )-conforming lowest-order Raviart-Thomas elements (face elements, Whitney 2-forms, [10, Sect. 3.2]), respectively. Recall that these spaces possess localized nodal bases whose functions are associated with vertices, edges, and faces of $\Omega_{l}$, respectively, and that form $L_{2}$-Riesz bases [26, (3.37)]. A tilde will tag subspaces of these finite element spaces comprising functions whose (appropriate) traces vanish on $\Gamma \backslash \Gamma^{+}$, i.e. $\widetilde{\mathcal{S}}_{1}\left(\Omega_{l}\right), \widetilde{\mathcal{N D}}_{1}\left(\Omega_{l}\right)$, and $\widetilde{\mathcal{R T}}_{0}\left(\Omega_{l}\right)$, accordingly. The $\sim$ symbol will also distinguish functions in these subspaces.

Standard boundary element (BE) spaces on $\Gamma_{l}$ arise from taking the natural traces of finite element functions on $\Omega_{l}, l \in \mathbb{N}_{0}$; see [29, Sect. 3]:

$$
\left\{\begin{aligned}
\mathcal{S}_{1}\left(\Gamma_{l}\right) & :=\mathrm{T}_{\boldsymbol{x}}\left(\mathcal{S}_{1}\left(\Omega_{l}\right)\right) \subset H^{\frac{1}{2}}(\Gamma):=\mathrm{T}_{\boldsymbol{x}}\left(H^{1}(\Omega)\right) \\
\mathcal{R}_{0}\left(\Gamma_{l}\right) & :=\mathrm{T}_{\boldsymbol{t}}\left(\mathcal{N} \mathcal{D}_{1}\left(\Omega_{l}\right)\right) \subset \boldsymbol{H}^{-\frac{1}{2}}\left(\operatorname{div}_{\Gamma}, \Gamma\right):=\mathrm{T}_{\boldsymbol{t}}(\boldsymbol{H}(\operatorname{curl}, \Omega)), \\
\mathcal{Q}_{0}\left(\Gamma_{l}\right) & :=\mathrm{T}_{\boldsymbol{n}}\left(\mathcal{R}_{0}\left(\Omega_{l}\right)\right) \subset H^{-\frac{1}{2}}(\Gamma):=\mathrm{T}_{\boldsymbol{n}}(\boldsymbol{H}(\operatorname{div}, \Omega))
\end{aligned}\right.
$$

where $\mathcal{Q}_{0}\left(\Gamma_{h}\right)$ is the space of piecewise constant discontinuous functions on $\Gamma_{h}$. Analogous to (2.4) we obtain "localized boundary element spaces":

$$
\widetilde{\mathcal{S}}_{1}\left(\Gamma_{l}^{+}\right):=\mathrm{T}_{\boldsymbol{x}}\left(\widetilde{\mathcal{S}}_{1}\left(\Omega_{l}\right)\right), \widetilde{\mathcal{R}}_{0}\left(\Gamma_{l}^{+}\right):=\mathrm{T}_{\boldsymbol{t}}\left(\widetilde{\mathcal{N}}_{1}\left(\Omega_{l}\right)\right), \widetilde{\mathcal{Q}}_{0}\left(\Gamma_{l}^{+}\right):=\mathrm{T}_{\boldsymbol{n}}\left(\widetilde{\mathcal{R}}_{0}\left(\Omega_{l}\right)\right) .
$$

These can be regarded as boundary element spaces on the sub-meshes $\Gamma_{l}^{+}:=\left.\Gamma_{l}\right|_{\Gamma^{+}}$ satisfying homogeneous boundary conditions on $\partial \Gamma^{+}$: functions in $\widetilde{\mathcal{S}}_{1}\left(\Gamma_{l}^{+}\right)$vanish on $\partial \Gamma^{+}$, whereas those in $\widetilde{\mathcal{R T}}_{0}\left(\Gamma_{l}^{+}\right)$feature zero (in plane) normal components on $\partial \Gamma^{+}$. Only for $\widetilde{\mathcal{Q}}_{0}\left(\Gamma_{l}^{+}\right)$no boundary conditions are implied and $\widetilde{\mathcal{Q}}_{0}\left(\Gamma_{l}^{+}\right)=\mathcal{Q}_{0}\left(\Gamma_{l}^{+}\right)$.

Slightly abusing notation, the localized boundary element spaces can be regarded as subspaces of localized trace spaces

$$
\widetilde{\mathcal{S}}_{1}\left(\Gamma_{l}^{+}\right) \subset \widetilde{H}^{\frac{1}{2}}\left(\Gamma^{+}\right), \quad \widetilde{\mathcal{R}}_{0}\left(\Gamma_{l}^{+}\right) \subset \widetilde{\boldsymbol{H}}^{-\frac{1}{2}}\left(\operatorname{div}_{\Gamma}, \Gamma^{+}\right), \quad \widetilde{\mathcal{Q}}_{0}\left(\Gamma_{l}^{+}\right) \subset \widetilde{H}^{-\frac{1}{2}}\left(\Gamma^{+}\right) .
$$

Here, following (1.2), the localized trace spaces comprise (generalized) functions on $\Gamma^{+}$, whose extension by zero to $\Gamma$ belong to the corresponding trace space on $\Gamma$ (see [36, Ch. 3], [11, Sect. 2.3]),

$$
\begin{aligned}
\widetilde{\boldsymbol{H}}^{-\frac{1}{2}}\left(\operatorname{div}_{\Gamma}, \Gamma^{+}\right) & :=\left\{\mathbf{u} \in \boldsymbol{H}^{-\frac{1}{2}}\left(\operatorname{div}_{\Gamma}, \Gamma^{+}\right),\|\mathbf{Z} \mathbf{u}\|_{\boldsymbol{H}^{-\frac{1}{2}}\left(\operatorname{div}_{\Gamma}, \Gamma\right)}<\infty\right\}, \\
\widetilde{H}^{-\frac{1}{2}}\left(\Gamma^{+}\right) & :=\left\{\varphi \in H^{-\frac{1}{2}}\left(\Gamma^{+}\right),\|\mathrm{Z} \varphi\|_{H^{-\frac{1}{2}}(\Gamma)}<\infty\right\},
\end{aligned}
$$

with the extension by zero operator $Z$ defined in (1.1). Norms on these spaces as well as on $\widetilde{H}^{\frac{1}{2}}\left(\Gamma^{+}\right)$can be computed by taking the trace norm on $\Gamma$ of the extensions by zero. The reader may imagine them as trace spaces on $\Gamma^{+}$with boundary conditions on $\partial \Gamma^{+}$though this is not the full picture. In order to fully understand the space $\widetilde{H}^{-\frac{1}{2}}(\Gamma)$, it is worth remembering the $L_{2}(\Gamma)$-dualities [28, Sect. 2.2]:

$$
\left(H^{\frac{1}{2}}\left(\Gamma^{+}\right)\right)^{\prime}=\widetilde{H}^{-\frac{1}{2}}\left(\Gamma^{+}\right) \subset H^{-\frac{1}{2}}\left(\Gamma^{+}\right)=\left(\widetilde{H}^{\frac{1}{2}}\left(\Gamma^{+}\right)\right)^{\prime} .
$$

Now we summarize the main results that extend (1.3) to the boundary element spaces $\widetilde{\mathcal{R T}}_{0}\left(\Gamma_{l}^{+}\right)$and $\mathcal{Q}_{0}\left(\Gamma^{+}\right)$and the corresponding (localized) trace norms.

Theorem 2.1. Under the assumptions on the families of meshes stated in Section 2, the following inverse inequality holds true for all $L \in \mathbb{N}_{0}$,

$$
\left\|\widetilde{\mathbf{v}}_{L}\right\|_{\widetilde{\boldsymbol{H}}^{-\frac{1}{2}}\left(\operatorname{div}_{\Gamma}, \Gamma^{+}\right)} \leq C\left(1+\left|\log h_{L}\right|^{\frac{3}{2}}\right)\left\|\widetilde{\mathbf{v}}_{L}\right\|_{\boldsymbol{H}^{-\frac{1}{2}}\left(\operatorname{div}_{\Gamma}, \Gamma^{+}\right)}, \quad \forall \widetilde{\mathbf{v}}_{L} \in \widetilde{\mathcal{R}}_{0}\left(\Gamma_{L}^{+}\right),
$$


where $C>0$ depends only on $\Omega, \Gamma^{+}$, and the shape-regularity of $\Omega_{0}$.

Theorem 2.2. In the setting detailed in Section 2 we find, with $C>0$ depending only on $\Omega, \Gamma^{+}$, and the shape-regularity of $\Omega_{0}$,

$$
\left\|\widetilde{\varphi}_{L}\right\|_{\widetilde{H}^{-\frac{1}{2}}\left(\Gamma^{+}\right)} \leq C\left(1+\left|\log h_{L}\right|^{\frac{3}{2}}\right)\left\|\widetilde{\varphi}_{L}\right\|_{H^{-\frac{1}{2}}\left(\Gamma^{+}\right)} \quad \forall \widetilde{\varphi}_{L} \in \widetilde{\mathcal{Q}}_{0}\left(\Gamma_{L}^{+}\right), \forall L \in \mathbb{N}_{0} .
$$

Remark 2.3. We do not claim that the bounds in Theorems 2.1 and 2.2 are sharp. It might be possible to decrease the powers of $\left|\log h_{L}\right|$ but this is left to future investigations; besides, numerical experiments are difficult to conduct, because they entail evaluation of localized trace norms. In addition, it is notoriously difficult to tell a power of $\left|\log h_{L}\right|$ from numerical measurements.

\section{Proof: CUt-off inequalities for $H^{\frac{1}{2}}$-CONFOrming BEM SPACES}

In this section we give a new proof of an inverse inequality related to (1.3), with a polylogarithmic bound $b(h)$. Admittedly, we merely recover results already used in domain decomposition theory. Yet, the point of this section is to elucidate the spirit of our approach in the relatively simple setting of $H^{\frac{1}{2}}(\Gamma)$ and $\mathcal{S}_{1}\left(\Gamma_{l}\right)$.

To begin with, we need uniformly stable discrete extension operators:

Lemma 3.1 (cf. [29, Lemma 5.1]). There exist extension operators $\mathrm{E}_{l}^{0}: \mathcal{S}_{1}\left(\Gamma_{l}\right) \rightarrow$ $\mathcal{S}_{1}\left(\Omega_{l}\right)$ and $\widetilde{\mathrm{E}}_{l}^{0}: \widetilde{\mathcal{S}}_{1}\left(\Gamma_{l}^{+}\right) \rightarrow \widetilde{\mathcal{S}}_{1}\left(\Omega_{l}\right)$ such that

$$
\mathrm{T}_{x} \mathrm{E}_{l}^{0} v_{l}=v_{l}, \quad \forall v_{l} \in \mathcal{S}_{1}\left(\Gamma_{l}\right), \quad \mathrm{T}_{x} \widetilde{\mathrm{E}}_{l}^{0} \widetilde{v}_{l}=\widetilde{v}_{l}, \quad \forall \widetilde{v}_{l} \in \widetilde{\mathcal{S}}_{1}\left(\Gamma_{l}^{+}\right),
$$

and they are uniformly stable in the sense that

$$
\begin{aligned}
& \left\|\mathrm{E}_{l}^{0} v_{l}\right\|_{H^{1}(\Omega)} \lesssim\left\|v_{l}\right\|_{H^{\frac{1}{2}}(\Gamma)}, \quad \forall v_{l} \in \mathcal{S}_{1}\left(\Gamma_{l}\right), \\
& \left\|\widetilde{\mathrm{E}}_{l}^{0} \widetilde{v}_{l}\right\|_{H^{1}(\Omega)} \lesssim\left\|\widetilde{v}_{l}\right\|_{\widetilde{H}^{\frac{1}{2}}\left(\Gamma^{+}\right)}, \quad \forall \widetilde{v}_{l} \in \widetilde{\mathcal{S}}_{1}\left(\Gamma_{l}^{+}\right) .
\end{aligned}
$$

Proof. The proof is standard employing harmonic extension and the famous ScottZhang quasi-interpolation operator [43]; see [47, Sect. 4.2.1 \& 4.2.2].

Another tool is given by the $L_{2}$-stable "nodal" extension operators:

Lemma 3.2. We can find uniformly stable extension operators $\mathrm{N}_{l}^{0}: \mathcal{S}_{1}\left(\Gamma_{l}\right) \rightarrow$ $\mathcal{S}_{1}\left(\Omega_{l}\right)$ and $\widetilde{\mathrm{N}}_{l}^{0}: \widetilde{\mathcal{S}}_{1}\left(\Gamma_{l}^{+}\right) \rightarrow \widetilde{\mathcal{S}}_{1}\left(\Omega_{l}\right)$ that satisfy

$$
\mathrm{T}_{x} \mathrm{~N}_{l}^{0} v_{l}=v_{l}, \quad \forall v_{l} \in \mathcal{S}_{1}\left(\Gamma_{l}\right), \quad \mathrm{T}_{x} \widetilde{\mathrm{N}}_{l}^{0} \widetilde{v}_{l}=\widetilde{v}_{l}, \quad \forall \widetilde{v}_{l} \in \widetilde{\mathcal{S}}_{1}\left(\Gamma_{l}^{+}\right),
$$

and

$$
\begin{aligned}
&\left\|\mathrm{N}_{l}^{0} v_{l}\right\|_{L_{2}(\Omega)} \lesssim h_{l}^{\frac{1}{2}}\left\|v_{l}\right\|_{L_{2}(\Gamma)}, \quad \forall v_{l} \in \mathcal{S}_{1}\left(\Gamma_{l}\right), \\
&\left\|\widetilde{\mathrm{N}}_{l}^{0} \widetilde{v}_{l}\right\|_{L_{2}(\Omega)} \lesssim h_{l}^{\frac{1}{2}}\left\|\widetilde{v}_{l}\right\|_{L_{2}(\Gamma)}, \quad \forall \widetilde{v}_{l} \in \widetilde{\mathcal{S}}_{1}\left(\Gamma_{l}^{+}\right) .
\end{aligned}
$$

Proof. Trivial discrete extension supplies both $\mathrm{N}_{l}^{0}$ and $\widetilde{\mathrm{N}}_{l}^{0}$. For instance,

$$
\left(\mathrm{N}_{l}^{0} v_{l}\right)(\boldsymbol{p}):= \begin{cases}v_{l}(\boldsymbol{p}), & \text { if } \boldsymbol{p} \in \mathcal{V}\left(\Omega_{l}\right) \cap \Gamma, \\ 0, & \text { if } \boldsymbol{p} \in \Omega .\end{cases}
$$

Then, simple scaling arguments bear out the estimates of the lemma. 
A crucial ingredient for our theory are multilevel norm equivalences, which arise from profound stability results about multilevel decompositions of finite element spaces. For the case of linear Lagrangian finite elements the relevant multilevel norms read for $V_{L} \in \mathcal{S}_{1}\left(\Omega_{L}\right), L \in \mathbb{N}_{0}$,

$$
\left\|V_{L}\right\|_{\mathcal{S}_{1}\left(\Omega_{L}\right)}^{2}:=\inf \left\{\sum_{l=0}^{L} h_{l}^{-2}\left\|W_{l}\right\|_{L_{2}(\Omega)}^{2}: V_{L}=\sum_{l=0}^{L} W_{l}, W_{l} \in \mathcal{S}_{1}\left(\Omega_{l}\right)\right\}
$$

and for $\widetilde{V}_{L} \in \widetilde{\mathcal{S}}_{1}\left(\Omega_{L}\right)$,

$$
\left\|\widetilde{V}_{L}\right\|_{\widetilde{\mathcal{S}}_{1}\left(\Omega_{L}\right)}^{2}:=\inf \left\{\sum_{l=0}^{L} h_{l}^{-2}\left\|\widetilde{W}_{l}\right\|_{L_{2}(\Omega)}^{2}: \widetilde{V}_{L}=\sum_{l=0}^{L} \widetilde{W}_{l}, \widetilde{W}_{l} \in \widetilde{\mathcal{S}}_{1}\left(\Omega_{l}\right)\right\} .
$$

The key result obtained in the study of multilevel preconditioners is the uniform equivalence of the multilevel norms and the $H^{1}(\Omega)$-norm on the finite element spaces. This was first proved in [40] and [49, and is further elaborated in [7, [41, Ch. 4], [46, Appendix], [8, and [30, Sect. 5].

Theorem 3.3 (see [30, Sect. 5]). The following norms are equivalent:

$$
\begin{aligned}
\left\|V_{L} \mid\right\|_{\mathcal{S}_{1}\left(\Omega_{L}\right)} \approx\left\|V_{L}\right\|_{H^{1}(\Omega)}, & \forall V_{L} \in \mathcal{S}_{1}\left(\Omega_{L}\right), \\
\left\|\widetilde{V}_{L}\right\|_{\widetilde{\mathcal{S}}_{1}\left(\Omega_{L}\right)} \approx\left\|\widetilde{V}_{L}\right\|_{H^{1}(\Omega)}, & \forall \widetilde{V}_{L} \in \widetilde{\mathcal{S}}_{1}\left(\Omega_{L}\right) .
\end{aligned}
$$

Similar norm equivalences also hold for boundary element spaces and involve the multilevel norms

$$
\left\|v_{L}\right\|_{\mathcal{S}_{1}\left(\Gamma_{L}\right)}^{2}:=\inf \left\{\sum_{l=0}^{L} h_{l}^{-1}\left\|w_{l}\right\|_{L_{2}(\Gamma)}^{2}: v_{L}=\sum_{l=0}^{L} w_{l}, w_{l} \in \mathcal{S}_{1}\left(\Gamma_{l}\right)\right\},
$$

for $v_{L} \in \mathcal{S}_{1}\left(\Gamma_{L}^{+}\right), L \in \mathbb{N}_{0}$, and

$$
\left\|\widetilde{v}_{L}\right\|_{\widetilde{\mathcal{S}}_{1}\left(\Gamma_{L}^{+}\right)}^{2}:=\inf \left\{\sum_{l=0}^{L} h_{l}^{-1}\left\|\widetilde{w}_{l}\right\|_{L_{2}(\Gamma)}^{2}: \widetilde{v}_{L}=\sum_{l=0}^{L} \widetilde{w}_{l}, \widetilde{w}_{l} \in \widetilde{\mathcal{S}}_{1}\left(\Gamma_{l}^{+}\right)\right\},
$$

for $\widetilde{v}_{L} \in \widetilde{\mathcal{S}}_{1}\left(\Gamma_{L}^{+}\right)$. Please note the different powers of the mesh widths, $h_{l}^{-2}$ versus $h_{l}^{-1}$, are used as weights in (3.1)-(3.2) and (3.3)-(3.4).

Theorem 3.4. On $H^{\frac{1}{2}}(\Gamma)$-conforming boundary element spaces we have the following equivalent norms:

$$
\begin{aligned}
\left\|v_{L}\right\|\left\|_{\mathcal{S}_{1}\left(\Gamma_{L}\right)} \approx\right\| v_{L} \|_{H^{\frac{1}{2}}(\Gamma)} & \forall v_{L} \in \mathcal{S}_{1}\left(\Gamma_{L}\right), \\
\left\|\widetilde{v}_{L} \mid\right\|_{\widetilde{\mathcal{S}}_{1}\left(\Gamma_{L}^{+}\right)} \approx\left\|\widetilde{v}_{L}\right\|_{\widetilde{H}^{\frac{1}{2}}\left(\Gamma^{+}\right)} & \forall \widetilde{v}_{L} \in \widetilde{\mathcal{S}}_{1}\left(\Gamma_{L}^{+}\right) .
\end{aligned}
$$

Proof. Proofs of (3.5) can be found in 2,23 for a more general setting. Here we rely on Oswald's idea [15, which is also discussed in [29, Sect. 2], and transfer the stability result of Theorem 3.3 to the boundary element spaces.

We restrict ourselves to the (more difficult) norm equivalence (3.6) on $\widetilde{\mathcal{S}}_{1}\left(\Gamma_{L}^{+}\right)$ and pick an arbitrary $\widetilde{v}_{L} \in \widetilde{\mathcal{S}}_{1}\left(\Gamma_{L}^{+}\right)$. By similar reasoning we can infer (3.5). The proof can be split into two steps: 
(1 First, we tackle the "¿" estimate. We consider the discrete extension $\widetilde{\mathrm{E}}_{L}^{0} \widetilde{v}_{L} \in$ $\widetilde{\mathcal{S}}_{1}\left(\Omega_{L}\right)$ from Lemma 3.1 and apply the result of Theorem 3.3 to $\widetilde{\mathrm{E}}_{L}^{0} \widetilde{v}_{L}$, which gives $\widetilde{W}_{l} \in \widetilde{\mathcal{S}}_{1}\left(\Omega_{l}\right), l=0, \ldots, L$, such that

$$
\sum_{l=0}^{L} \widetilde{W}_{l}=\widetilde{\mathrm{E}}_{L}^{0} \widetilde{v}_{L}, \quad \sum_{l=0}^{L} h_{l}^{-2}\left\|\widetilde{W}_{l}\right\|_{L_{2}(\Omega)}^{2} \lesssim\left\|\widetilde{\mathrm{E}}_{L}^{0} \widetilde{v}_{L}\right\|_{H^{1}(\Omega)}^{2} \lesssim\left\|\widetilde{v}_{L}\right\|_{\widetilde{H}^{\frac{1}{2}}\left(\Gamma^{+}\right)}^{2} .
$$

We define $\widetilde{w}_{l}:=\mathrm{T}_{\boldsymbol{x}} \widetilde{W}_{l} \in \widetilde{\mathcal{S}}_{1}\left(\Gamma_{l}^{+}\right)$and find

$$
\begin{aligned}
& \sum_{l=0}^{L} \widetilde{w}_{l}=\sum_{l=0}^{L} \mathrm{~T}_{\boldsymbol{x}} \widetilde{W}_{l}=\mathrm{T}_{\boldsymbol{x}} \widetilde{\mathrm{E}}_{L}^{0} \widetilde{v}_{L}=\widetilde{v}_{L}, \\
& \sum_{l=0}^{L} h_{l}^{-1}\left\|\widetilde{w}_{l}\right\|_{L_{2}(\Gamma)}^{2} \stackrel{(*)}{\lesssim} \sum_{l=0}^{L} h_{l}^{-2}\left\|\widetilde{W}_{l}\right\|_{L_{2}(\Omega)}^{2} \lesssim\left\|\widetilde{\mathrm{E}}_{L}^{0} \widetilde{v}_{L}\right\|_{H^{1}(\Omega)}^{2} \lesssim\left\|\widetilde{v}_{L}\right\|_{\widetilde{H}^{\frac{1}{2}}\left(\Gamma^{+}\right)}^{2},
\end{aligned}
$$

where the estimate $(*)$ is a consequence of the simple scaling inequality

$$
\left\|\mathrm{T}_{\boldsymbol{x}} V_{l}\right\|_{L_{2}(\Gamma)} \lesssim h_{l}^{-\frac{1}{2}}\left\|V_{l}\right\|_{L_{2}(\Omega)}, \quad \forall V_{l} \in \mathcal{S}_{1}\left(\Omega_{l}\right) .
$$

Thus, in (3.7) we have found a candidate splitting of $\widetilde{v}_{L}$, which realizes the estimate " $\lesssim$ " claimed in the theorem.

(2) Now, we establish the estimate "邓". Pick arbitrary $\widetilde{w}_{l} \in \widetilde{\mathcal{S}}_{1}\left(\Gamma_{l}^{+}\right)$that add up to $\widetilde{v}_{L}$, i.e.,

$$
\sum_{l=0}^{L} \widetilde{w}_{l}=\widetilde{v}_{L}
$$

By trivial extension according to Lemma 3.2 , we obtain $\widetilde{W}_{l}:=\widetilde{\mathrm{N}}_{l}^{0} \widetilde{w}_{l} \in \widetilde{\mathcal{S}}_{1}\left(\Omega_{l}\right)$, with the property

$$
\mathrm{\top}_{\boldsymbol{x}} \widetilde{V}_{L}=\widetilde{v}_{L} \quad \text { for } \quad \widetilde{V}_{L}:=\sum_{l=0}^{L} \widetilde{W}_{l} \in \widetilde{\mathcal{S}}_{1}\left(\Omega_{L}\right) .
$$

By continuity of the trace operator $\mathrm{T}_{\boldsymbol{x}}$, it holds that

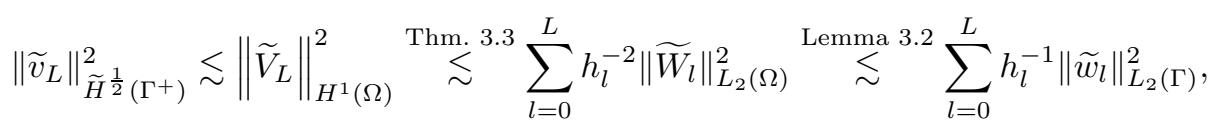

which concludes the proof.

The localized trace norm of boundary element functions also allows an equivalent characterization through multilevel splittings:

$$
\left\|v_{L}\right\|_{\mathcal{S}_{1}\left(\Gamma_{L}^{+}\right)}^{2}:=\inf \left\{\sum_{l=0}^{L} h_{l}^{-1}\left\|w_{l}\right\|_{L_{2}(\Gamma)}^{2}: v_{L}=\left.\left(\sum_{l=0}^{L} w_{l}\right)\right|_{\Gamma^{+}}, w_{l} \in \mathcal{S}_{1}\left(\Gamma_{l}\right)\right\} .
$$

The precise statement is made in the next lemma. At this point note that the norms for the terms in the splitting for (3.9) are computed on the entire boundary $\Gamma$. In addition, we note that we deal with three different multilevel norms $\|\cdot \mid\|_{\mathcal{S}_{1}\left(\Gamma_{L}\right)}$, $\|\mid\| \|_{\widetilde{\mathcal{S}}_{1}\left(\Gamma_{L}^{+}\right)}$, and $\|\cdot \mid\|_{\mathcal{S}_{1}\left(\Gamma_{L}^{+}\right)}$.

Lemma 3.5. On $\mathcal{S}_{1}\left(\Gamma_{L}^{+}\right)$we have equivalence of norms according to

$$
\left\|v_{L}\right\|_{H^{\frac{1}{2}\left(\Gamma^{+}\right)}} \approx\left\|\mid v_{L}\right\|_{\mathcal{S}_{1}\left(\Gamma_{L}^{+}\right)} \quad \forall v_{L} \in \mathcal{S}_{1}\left(\Gamma_{L}^{+}\right) .
$$


Proof. By the standard definition of Sobolev norms on sub-domains and the continuity of the trace operator, we obtain

$$
\begin{aligned}
\left\|v_{L}\right\|_{H^{\frac{1}{2}}\left(\Gamma^{+}\right)} & \approx \inf \left\{\|\widehat{v}\|_{H^{\frac{1}{2}}(\Gamma)}: \widehat{v} \in H^{\frac{1}{2}}(\Gamma),\left.\widehat{v}\right|_{\Gamma^{+}}=v_{L}\right\} \\
& \approx \inf \left\{\|\widehat{V}\|_{H^{1}(\Omega)}: \widehat{V} \in H^{1}(\Omega),\left.\mathrm{\top}_{\boldsymbol{x}} \widehat{V}\right|_{\Gamma^{+}}=v_{L}\right\} .
\end{aligned}
$$

Next, we take the cue from the proof of Lemma 3.1 and apply Scott-Zhang quasiinterpolation to $\widehat{V}$. We make use of the fact that $\overline{\Gamma^{+}}$is a union of (closed) triangles on all levels. Thus, the continuity of quasi-interpolation in $H^{1}(\Omega)$ and the preservation of boundary values on $\Gamma^{+}$permit us to conclude

$$
\begin{aligned}
\left\|v_{L}\right\|_{H^{\frac{1}{2}}\left(\Gamma^{+}\right)} & \approx \inf \left\{\left\|\widehat{V}_{L}\right\|_{H^{1}(\Omega)}: \widehat{V}_{L} \in \mathcal{S}_{1}\left(\Omega_{L}\right),\left.\mathrm{T}_{\boldsymbol{x}} \widehat{V}_{L}\right|_{\Gamma^{+}}=v_{L}\right\}, \\
& \approx \inf \left\{\left\|\widehat{v}_{L}\right\|_{H^{\frac{1}{2}(\Gamma)}}: \widehat{v}_{L} \in \mathcal{S}_{1}\left(\Gamma_{L}\right),\left.\widehat{v}_{L}\right|_{\Gamma^{+}}=v_{L}\right\} .
\end{aligned}
$$

To finish the proof we appeal to the estimate from Theorem 3.4

Now we are in position to prove our version of the "face lemmas" [45, Lemma 4.26] and [47, Lemma 4.10] for piecewise linear continuous boundary element spaces. We rely on a nodal cut-off $2^{2}$ operator $Z_{l}^{0}$ that sets a function to zero in all vertices of $\Gamma_{l}$ outside $\Gamma^{+}$. Concretely, on $\mathcal{S}_{1}\left(\Gamma_{l}\right)$ this cut-off is defined by

$$
\mathrm{Z}_{l}^{0} v_{l} \in \widetilde{\mathcal{S}}_{1}\left(\Gamma_{l}^{+}\right): \quad\left(\mathrm{Z}_{l}^{0} v_{l}\right)(\boldsymbol{p})= \begin{cases}v_{l}(\boldsymbol{p}), & \text { for } \boldsymbol{p} \in \mathcal{V}\left(\Gamma_{l}\right) \cap \Gamma^{+}, \\ 0, & \text { for } \boldsymbol{p} \in \mathcal{V}\left(\Gamma_{l}\right), \boldsymbol{p} \notin \Gamma^{+}\end{cases}
$$

Theorem 3.6. Under the assumptions on the families of meshes stated in Section 2 the following inverse inequality holds true:

$$
\left\|\mathrm{Z}_{L}^{0} v_{L}\right\|_{\widetilde{H}^{\frac{1}{2}}\left(\Gamma^{+}\right)} \lesssim\left(1+\left|\log h_{L}\right|\right)\left\|v_{L}\right\|_{H^{\frac{1}{2}}\left(\Gamma^{+}\right)}, \quad \forall v_{L} \in \mathcal{S}_{1}\left(\Gamma_{L}^{+}\right) .
$$

Proof. We start with the crucial observation, owing to Theorem 3.4 and Lemma 3.5. that the proof boils down to showing

$$
\left\|\mathrm{Z}_{L}^{0} v_{L}\right\|_{\widetilde{\mathcal{S}}_{1}\left(\Gamma_{L}^{+}\right)}^{2} \lesssim L^{2}\left\|v_{L}\right\|_{\mathcal{S}_{1}\left(\Gamma_{L}^{+}\right)}^{2},
$$

where the multilevel norm on the left-hand side is defined in (3.4) while that on the right-hand side is from (3.9). Fix $v_{L} \in \mathcal{S}_{1}\left(\Gamma_{L}^{+}\right)$and pick arbitrary $\widehat{w}_{l} \in \mathcal{S}_{1}\left(\Gamma_{l}\right)$ such that

$$
v_{L}=\left.\left(\sum_{l=0}^{L} \widehat{w}_{l}\right)\right|_{\Gamma^{+}}
$$

This will play the role of the minimizing multilevel decomposition on the right-hand side of (3.11). To establish the assertion of the theorem we have to show that we can find $\widetilde{w}_{l} \in \widetilde{\mathcal{S}}_{1}\left(\Gamma_{l}^{+}\right)$with

$$
\mathrm{Z}_{L}^{0} v_{L}=\sum_{l=0}^{L} \widetilde{w}_{l}
$$

such that

$$
\sum_{l=0}^{L} h_{l}^{-1}\left\|\widetilde{w}_{l}\right\|_{L_{2}(\Gamma)}^{2} \lesssim L^{2} \sum_{l=0}^{L} h_{l}^{-1}\left\|\widehat{w}_{l}\right\|_{L_{2}\left(\Gamma^{+}\right)}^{2}
$$

\footnotetext{
${ }^{2}$ For the cut-off operator 47] uses the notation $I_{\Gamma}^{0}$, whereas [45] writes $I^{h}$.
} 
The construction of the $\widetilde{w}_{l}$ starts with the accumulation of multilevel contributions to $v_{L}$ :

$$
\widehat{q}_{l}:=\sum_{k=0}^{l} \widehat{w}_{k} \in \mathcal{S}_{1}\left(\Gamma_{l}\right), \quad l=0, \ldots, L,
$$

which then undergo the cut-off to zero,

$$
\widetilde{q}_{l}=\mathrm{Z}_{l}^{0} \widehat{q}_{l} \in \widetilde{\mathcal{S}}_{1}\left(\Gamma_{l}^{+}\right), \quad l=0, \ldots, L .
$$

The next steps are inspired by a trick invented by P. Oswald in [39, Cor. 30]. We define

$$
\widetilde{w}_{l}:=\widetilde{q}_{l}-\widetilde{q}_{l-1} \in \widetilde{\mathcal{S}}_{1}\left(\Gamma_{l}^{+}\right), \quad l=1, \ldots, L, \quad \widetilde{w}_{0}=\widetilde{q}_{0}=\mathrm{Z}_{0}^{0} \widehat{w}_{0} \in \widetilde{\mathcal{S}}_{1}\left(\Gamma_{0}^{+}\right) .
$$

Setting $\widetilde{q}_{-1}:=0$ we can rewrite

$$
\begin{aligned}
\widetilde{w}_{l} & =\left(\widetilde{q}_{l}-\widetilde{q}_{l-1}\right)-\left(\widehat{q}_{l}-\widehat{q}_{l-1}\right)+\widehat{w}_{l}, \\
& =\left(\widetilde{q}_{l}-\widehat{q}_{l}\right)-\left(\widetilde{q}_{l-1}-\widehat{q}_{l-1}\right)+\widehat{w}_{l} .
\end{aligned}
$$

Hence, in order to deal with the left-hand side of (3.14) we have to estimate $\left\|\widetilde{q}_{l}-\widehat{q}_{l}\right\|_{L_{2}\left(\Gamma^{+}\right)}=\left\|\left(Z_{l}^{0}-\mathrm{Id}\right) \widehat{q}_{l}\right\|_{L_{2}\left(\Gamma^{+}\right)}$. Notice that on $\Gamma^{+}$only basis functions located on the boundary $\partial \Gamma^{+}$contribute to $\left(Z_{l}^{0}-\mathrm{Id}\right) \widehat{q}_{l}$. Also recall, the uniform $L_{2}$-stability of the nodal bases, from which we conclude by elementary scaling arguments that

$$
\begin{aligned}
\left\|\widetilde{q}_{l}-\widehat{q}_{l}\right\|_{L_{2}\left(\Gamma^{+}\right)}^{2} & \lesssim h_{l}\left\|\widehat{q}_{l}\right\|_{L_{2}\left(\partial \Gamma^{+}\right)}^{2} \lesssim h_{l}\left\|\sum_{k=0}^{l} \widehat{w}_{k}\right\|_{L_{2}\left(\partial \Gamma^{+}\right)}^{2} \\
& \lesssim h_{l}\left(\sum_{k=0}^{l}\left\|\widehat{w}_{k}\right\|_{L_{2}\left(\partial \Gamma^{+}\right)}\right)^{2} \lesssim h_{l}\left(\sum_{k=0}^{l} h_{k}^{-\frac{1}{2}}\left\|\widehat{w}_{k}\right\|_{L_{2}\left(\Gamma^{+}\right)}\right)^{2} \\
& \lesssim h_{l} l \cdot \sum_{k=0}^{l} h_{k}^{-1}\left\|\widehat{w}_{k}\right\|_{L_{2}\left(\Gamma^{+}\right)}^{2}
\end{aligned}
$$

by using the Cauchy-Schwarz inequality on $\mathbb{R}^{l+1}$ in the last step. Simple rearrangement of summations shows (3.14),

$$
\begin{aligned}
\sum_{l=0}^{L} h_{l}^{-1}\left\|\widetilde{w}_{l}\right\|_{L_{2}(\Gamma)}^{2} & \lesssim \sum_{l=0}^{L} h_{l}^{-1}\left(\left\|\widetilde{q}_{l}-\widehat{q}_{l}\right\|_{L_{2}\left(\Gamma^{+}\right)}^{2}+\left\|\widehat{w}_{l}\right\|_{L_{2}\left(\Gamma^{+}\right)}^{2}\right) \\
& \stackrel{\sqrt{3.19}}{\lesssim} \sum_{l=0}^{L} l \sum_{k=0}^{l} h_{k}^{-1}\left\|\widehat{w}_{k}\right\|_{L_{2}\left(\Gamma^{+}\right)}^{2}+\sum_{l=0}^{L} h_{l}^{-1}\left\|\widehat{w}_{l}\right\|_{L_{2}\left(\Gamma^{+}\right)}^{2} \\
& \lesssim L^{2} \sum_{k=0}^{L} h_{k}^{-1}\left\|\widehat{w}_{k}\right\|_{L_{2}\left(\Gamma^{+}\right)}^{2}
\end{aligned}
$$

thereby finishing the proof.

Obviously, in our setting, (1.3) is a corollary of Theorem 3.6. Of course, we could have stated the theorem like Theorem 2.1. namely for functions in $\widetilde{\mathcal{S}}_{1}\left(\Gamma_{L}^{+}\right)$ dispensing with the cut-off operator $Z_{L}^{0}$. Yet, we have come to the conclusion that 
it is illuminating to grasp what prevents us from achieving a cut-off inverse estimate analogous to that of Theorem 3.6]also for $\boldsymbol{H}^{-\frac{1}{2}}\left(\operatorname{div}_{\Gamma}, \Gamma\right)$-conforming boundary elements. This will be discussed in Remark 6.1 in Section 6.

Remark 3.7. A closer scrutiny of results on multilevel decompositions for $H^{1}(\Omega)$ conforming finite element spaces [41, Sect. 4.2.2] and of the arguments of this section reveals that Theorem 3.6 remains valid in settings where the meshes $\Omega_{l}$ arise from local refinement.

\section{Discrete EXtension operators}

The stable discrete extension from Lemma 3.1 played a key role in the proof of the multilevel decomposition Theorem 3.4 for $H^{\frac{1}{2}}(\Gamma)$-conforming boundary element spaces. This section is dedicated to the counterparts of Lemmas 3.1 and 3.2 for $\boldsymbol{H}^{-\frac{1}{2}}\left(\operatorname{div}_{\Gamma}, \Gamma\right)$ - and $H^{-\frac{1}{2}}(\Gamma)$-conforming boundary element spaces $\mathcal{R} \mathcal{T}_{0}\left(\Gamma_{l}\right)$ and $\mathcal{Q}_{0}\left(\Gamma_{l}\right)$.

To begin with, the very analogues of the trivial nodal extensions that underly Lemma 3.2 also work for the other finite element spaces.

Lemma 4.1. There are extension operators $\mathrm{N}_{l}^{1}: \mathcal{R} \mathcal{T}_{0}\left(\Gamma_{l}\right) \rightarrow \mathcal{N} \mathcal{D}_{1}\left(\Omega_{l}\right)$ and $\widetilde{\mathrm{N}}_{l}^{1}$ : $\widetilde{\mathcal{R T}}_{0}\left(\Gamma_{l}^{+}\right) \rightarrow \widetilde{\mathcal{N D}}_{1}\left(\Omega_{l}\right)$ that satisfy

$$
\mathrm{T}_{\boldsymbol{t}}\left(\mathrm{N}_{l}^{1} \mathbf{v}_{l}\right)=\mathbf{v}_{l}, \quad \forall \mathbf{v}_{l} \in \mathcal{R} \mathcal{T}_{0}\left(\Gamma_{l}\right), \quad \mathrm{T}_{\boldsymbol{t}}\left(\widetilde{\mathrm{N}}_{l}^{1} \widetilde{\mathbf{v}}_{l}\right)=\widetilde{\mathbf{v}}_{l}, \quad \forall \widetilde{\mathbf{v}}_{l} \in \widetilde{\mathcal{R}}_{0}\left(\Gamma_{l}^{+}\right),
$$

and

$$
\begin{aligned}
&\left\|\mathrm{N}_{l}^{1} \mathbf{v}_{l}\right\|_{L_{2}(\Omega)} \lesssim h_{l}^{\frac{1}{2}}\left\|\mathbf{v}_{l}\right\|_{L_{2}(\Gamma)}, \quad \forall \mathbf{v}_{l} \in \mathcal{R} \mathcal{T}_{0}\left(\Gamma_{l}\right) \\
&\left\|\widetilde{N}_{l}^{1} \widetilde{\mathbf{v}}_{l}\right\|_{L_{2}(\Omega)} \lesssim h_{l}^{\frac{1}{2}}\left\|\widetilde{\mathbf{v}}_{l}\right\|_{L_{2}(\Gamma)}, \quad \forall \widetilde{\mathbf{v}}_{l} \in \widetilde{\mathcal{R}}_{0}\left(\Gamma_{l}^{+}\right)
\end{aligned}
$$

Proof. Choose both $\mathrm{N}_{l}^{1}$ and $\widetilde{\mathrm{N}}_{l}^{1}$ as trivial nodal extensions. Then use scaling arguments; see [29, Sect. 5.2] for details.

Lemma 4.2. There are extension operators $\mathrm{N}_{l}^{2}: \mathcal{Q}_{0}\left(\Gamma_{l}\right) \rightarrow \mathcal{R} \mathcal{T}_{0}\left(\Omega_{l}\right)$ and $\widetilde{\mathrm{N}}_{l}^{2}$ : $\widetilde{\mathcal{Q}}_{0}\left(\Gamma_{l}^{+}\right) \rightarrow \widetilde{\mathcal{R}}_{0}\left(\Omega_{l}\right)$ that satisfy

$$
\mathrm{T}_{\boldsymbol{t}}\left(\mathrm{N}_{l}^{2} \varphi_{l}\right)=\varphi_{l}, \quad \forall \varphi_{l} \in \mathcal{Q}_{0}\left(\Gamma_{l}\right), \quad \mathrm{T}_{\boldsymbol{t}}\left(\widetilde{\mathrm{N}}_{l}^{2} \widetilde{\varphi}_{l}\right)=\widetilde{\varphi}_{l}, \quad \forall \widetilde{\varphi}_{l} \in \widetilde{\mathcal{Q}}_{0}\left(\Gamma_{l}^{+}\right),
$$

and

$$
\begin{aligned}
&\left\|\mathrm{N}_{l}^{2} \varphi_{l}\right\|_{L_{2}(\Omega)} \lesssim h_{l}^{\frac{1}{2}}\left\|\varphi_{l}\right\|_{L_{2}(\Gamma)}, \quad \forall \varphi_{l} \in \mathcal{Q}_{0}\left(\Gamma_{l}\right), \\
&\left\|\widetilde{\mathrm{N}}_{l}^{2} \widetilde{\varphi}_{l}\right\|_{L_{2}(\Omega)} \lesssim h_{l}^{\frac{1}{2}}\left\|\widetilde{\varphi}_{l}\right\|_{L_{2}(\Gamma)}, \quad \forall \widetilde{\varphi}_{l} \in \widetilde{\mathcal{Q}}_{0}\left(\Gamma_{l}^{+}\right) .
\end{aligned}
$$

Proof. Again, use trivial nodal extension and appeal to scaling arguments as in [29, Sect. 5.2].

Surprisingly, the corresponding stable extensions are by no means standard and require sophisticated techniques; finding a stable discrete extension for surface edge elements $\boldsymbol{\mathcal { R }} \mathcal{T}_{0}\left(\Gamma_{l}\right)$ was one of the main challenges encountered in [29] and [3. Sect. 3], and our approach here closely follows [29, Sect. 5].

Outside of the $H^{1}(\Omega)-H^{\frac{1}{2}}(\Gamma)$ setting treated in Section 3. difficulties are compounded by the lack of Scott-Zhang type quasi-interpolation operators onto $\mathcal{N D}_{1}\left(\Omega_{h}\right)$ and $\mathcal{R} \mathcal{T}_{0}\left(\Omega_{h}\right)$ that preserve boundary values; cf. [16. Instead we have 
to resort to the nodal projectors $\Pi_{1}^{\mathrm{Rt}}$ onto $\mathcal{R} \mathcal{T}_{0}\left(\Omega_{l}\right)$ that are based on the normal fluxes through the faces of the tetrahedral mesh $\Omega_{l}$ - the degrees of freedom for $\boldsymbol{\mathcal { R }} \mathcal{T}_{0}\left(\Omega_{l}\right)$. From [26, Sect. 3.2] remember the crucial commuting diagram property

$$
\operatorname{div} \circ \Pi_{1}^{\mathrm{Rt}}=\Pi_{1}^{\mathrm{Q}} \circ \operatorname{div},
$$

for sufficiently smooth functions, where $\Pi_{1}^{\mathrm{Q}}$ is the $L_{2}(\Omega)$-orthogonal projection onto the space of piecewise constant functions on $\Omega_{l}$. Further, recall a special version of the interpolation error bound from [26, Thm. 3.16]:

Lemma 4.3. For any $\epsilon \in\left(0, \frac{1}{2}\right]$ the nodal interpolation operators $\Pi_{1}^{\mathrm{Rt}}:\left(C^{\infty}(\bar{\Omega})\right)^{3} \rightarrow$ $\mathcal{R T}_{0}\left(\Omega_{l}\right)$ can be extended to continuous mappings $\Pi_{1}^{\mathrm{Rt}}:\left\{\mathbf{V} \in\left(H^{\epsilon}(\Omega)\right)^{3}: \operatorname{div} \mathbf{V}=\right.$ $0\} \rightarrow\left\{\mathbf{V}_{l} \in \mathcal{R} \mathcal{T}_{0}\left(\Omega_{l}\right): \operatorname{div} \mathbf{V}_{l}=0\right\}$ that satisfy the interpolation error estimate

$$
\left\|\mathbf{V}-\Pi_{1}^{\mathrm{Rt}} \mathbf{V}\right\|_{L_{2}(\Omega)} \lesssim h_{l}^{\epsilon}\|\mathbf{V}\|_{H^{\epsilon}(\Omega)}, \quad \forall \mathbf{V} \in\left\{\mathbf{V} \in\left(H^{\epsilon}(\Omega)\right)^{3}: \operatorname{div} \mathbf{V}=0\right\}
$$

This estimate is instrumental in constructing various discrete extension operators for both boundary element spaces $\mathcal{Q}_{0}\left(\Gamma_{l}\right)$ and $\mathcal{R} \mathcal{T}_{0}\left(\Gamma_{l}\right)$. We start with the "simpler" case of $\mathcal{Q}_{0}\left(\Gamma_{l}\right)$, where the extension specifically maps into

$$
\boldsymbol{H}(\operatorname{div} 0, \Omega):=\{\mathbf{V} \in \boldsymbol{H}(\operatorname{div}, \Omega): \operatorname{div} \mathbf{V}=0\} .
$$

Lemma 4.4. We can find discrete extension operators $\mathrm{E}_{l}^{2}: \mathcal{Q}_{0}\left(\Gamma_{l}\right) \rightarrow \mathcal{R} \mathcal{T}_{0}\left(\Omega_{l}\right) \cap$ $\boldsymbol{H}(\operatorname{div} 0, \Omega), \widetilde{\mathrm{E}}_{l}^{2}: \widetilde{\mathcal{Q}}_{0}\left(\Gamma_{l}^{+}\right) \rightarrow \widetilde{\boldsymbol{\mathcal { T }}}_{0}\left(\Omega_{l}\right) \cap \boldsymbol{H}(\operatorname{div} 0, \Omega)$, and $\widehat{\mathrm{E}}_{l}^{2}: \mathcal{Q}_{0}\left(\Gamma_{l}^{+}\right) \rightarrow \boldsymbol{\mathcal { R }} \mathcal{T}_{0}\left(\Omega_{l}\right) \cap$ $\boldsymbol{H}(\operatorname{div} 0, \Omega)$ such that for all $l \in \mathbb{N}_{0}$,

$$
\begin{array}{ll}
\mathrm{T}_{n} \mathrm{E}_{l}^{2} \varphi_{l}=\varphi_{l}, & \text { on } \Gamma, \quad \forall \varphi_{l} \in \mathcal{Q}_{0}\left(\Gamma_{l}\right), \\
\mathrm{T}_{n} \widetilde{\mathrm{E}}_{l}^{2} \widetilde{\varphi}_{l}=\widetilde{\varphi}_{l}, & \text { on } \Gamma, \quad \forall \widetilde{\varphi}_{l} \in \widetilde{\mathcal{Q}}_{0}\left(\Gamma_{l}^{+}\right), \\
\mathrm{T}_{n} \widehat{\mathrm{E}}_{l}^{2} \widehat{\varphi}_{l}=\widehat{\varphi}_{l}, & \text { on } \Gamma^{+}, \quad \forall \widehat{\varphi}_{l} \in \mathcal{Q}_{0}\left(\Gamma_{l}^{+}\right),
\end{array}
$$

and they are uniformly stable in the sense that

$$
\begin{aligned}
& \left\|\mathrm{E}_{l}^{2} \varphi_{l}\right\|_{\boldsymbol{H}(\operatorname{div}, \Omega)} \lesssim\left\|\varphi_{l}\right\|_{H^{-\frac{1}{2}}(\Gamma)}, \quad \forall \varphi_{l} \in \mathcal{Q}_{0}\left(\Gamma_{l}\right), \forall l \in \mathbb{N}_{0}, \\
& \left\|\widetilde{\mathrm{E}}_{l}^{2} \widetilde{\varphi}_{l}\right\|_{\boldsymbol{H}(\operatorname{div}, \Omega)} \lesssim\left\|\widetilde{\varphi}_{l}\right\|_{H^{-\frac{1}{2}}(\Gamma)}, \quad \forall \widetilde{\varphi}_{l} \in \widetilde{\mathcal{Q}}_{0}\left(\Gamma_{l}^{+}\right), \forall l \in \mathbb{N}_{0}, \\
& \left\|\widehat{\mathrm{E}}_{l}^{2} \widehat{\varphi}_{l}\right\|_{\boldsymbol{H}(\operatorname{div}, \Omega)} \lesssim\left\|\widehat{\varphi}_{l}\right\|_{H^{-\frac{1}{2}}\left(\Gamma^{+}\right)}, \quad \forall \widehat{\varphi}_{l} \in \mathcal{Q}_{0}\left(\Gamma_{l}^{+}\right), \forall l \in \mathbb{N}_{0} .
\end{aligned}
$$

Proof. We restrict ourselves to $\widehat{\mathrm{E}}_{l}^{2}$, because the proofs for the other two extension operators are so similar that they can safely be left to the reader.

Pick $\widehat{\varphi}_{l} \in \mathcal{Q}_{0}\left(\Gamma_{l}^{+}\right)$and let $U$ be the solution of the boundary value problem

$$
\left\{\begin{aligned}
-\Delta U & =0, & & \text { in } \Omega, \\
\frac{\partial U}{\partial \boldsymbol{n}} & =\widehat{\varphi}_{l}, & & \text { on } \Gamma^{+}, \\
U & =0, & & \text { on } \Gamma \backslash \Gamma^{+} .
\end{aligned}\right.
$$

Thanks to an elliptic lifting result $[22$, Thms. 2.6.1, 2.6.3], there exists a unique solution $U \in H^{1+\epsilon}(\Omega)$ for any $\epsilon \in\left[0, \epsilon_{\Omega}\right)$ for some $\frac{1}{4}<\epsilon_{\Omega}<\frac{3}{4}$ that depends on $\Omega$ and $\Gamma^{+}$. Let us fix an eligible $\epsilon>\frac{1}{4}$, then

$$
\|U\|_{H^{1+\epsilon}(\Omega)} \lesssim\left\|\widehat{\varphi}_{l}\right\|_{H^{\epsilon-\frac{1}{2}}\left(\Gamma^{+}\right)} .
$$


Next, recall a slightly modified version of the inverse inequality of [29, Lemma 5.4]:

$$
\left\|\widehat{\nu}_{l}\right\|_{H^{\epsilon-\frac{1}{2}}\left(\Gamma^{+}\right)} \lesssim h_{l}^{-\epsilon}\left\|\widehat{\nu}_{l}\right\|_{H^{-\frac{1}{2}}\left(\Gamma^{+}\right)}, \quad \forall \nu_{l} \in \mathcal{Q}_{0}\left(\Gamma_{l}^{+}\right) .
$$

Its proof runs parallel to part (ii) of the proof of [29, Lemma 5.4].

Then we set $\mathbf{W}=\operatorname{grad} U$ and conclude $\mathbf{W} \in \boldsymbol{H}(\operatorname{div} 0, \Omega) \cap\left(H^{\epsilon}(\Omega)\right)^{3}$. Therefore, Lemma 4.3 implies for $\mathbf{W}_{l}:=\Pi_{1}^{\mathrm{Rt}} \mathbf{W}$ that

$$
\begin{aligned}
\left\|\mathbf{W}-\mathbf{W}_{l}\right\|_{L_{2}(\Omega)} & \lesssim h_{l}^{\epsilon}\|\mathbf{W}\|_{H^{\epsilon}(\Omega)} \lesssim h_{l}^{\epsilon}\|U\|_{H^{1+\epsilon}(\Omega)} \\
& \stackrel{(4.7)}{\lesssim} h_{l}^{\epsilon}\left\|\widehat{\varphi}_{l}\right\|_{H^{\epsilon-\frac{1}{2}}\left(\Gamma^{+}\right)} \stackrel{(4.8)}{\lesssim}\left\|\widehat{\varphi}_{l}\right\|_{H^{-\frac{1}{2}}\left(\Gamma^{+}\right)} .
\end{aligned}
$$

In addition, the commuting diagram property (4.1) ensures $\mathbf{W}_{l} \in \boldsymbol{H}(\operatorname{div} 0, \Omega)$ since

$$
\operatorname{div} \mathbf{W}_{l}=\operatorname{div} \Pi_{1}^{\mathrm{Rt}} \mathbf{W} \stackrel{4.11}{=} \Pi_{l}^{\mathrm{Q}} \operatorname{div} \mathbf{W}=0 .
$$

There is another commuting diagram property involving nodal projectors and trace operators

$$
\mathrm{T}_{\boldsymbol{n}} \circ \Pi_{1}^{\mathrm{Rt}}=\Pi_{1}^{\mathrm{Q}} \circ \mathrm{T}_{\boldsymbol{n}},
$$

for sufficiently smooth functions, where we slightly abused notation and still wrote $\Pi_{1}^{\mathrm{Q}}$ for the $L_{2}(\Gamma)$-orthogonal projection onto $\mathcal{Q}_{0}\left(\Gamma_{l}\right)$. The latter projection is completely local, which means

$\mathrm{T}_{\boldsymbol{n}} \mathbf{W}_{l}=\mathrm{T}_{\boldsymbol{n}} \Pi_{1}^{\mathrm{Rt}} \mathbf{W} \stackrel{(4.10)}{=} \Pi_{1}^{\mathrm{Q}}\left(\mathrm{T}_{\boldsymbol{n}} \mathbf{W}\right)=\Pi_{1}^{\mathrm{Q}}\left(\mathrm{T}_{\boldsymbol{n}} \operatorname{grad} U\right) \stackrel{(4.6)}{=} \Pi_{1}^{\mathrm{Q}}\left(\widehat{\varphi}_{l}\right)=\widehat{\varphi}_{l} \quad$ on $\Gamma^{+}$.

Eventually, we can set $\widehat{\mathrm{E}}_{l}^{2} \widehat{\varphi}_{l}:=\mathbf{W}_{l}$, because

$$
\left\|\mathbf{W}_{l}\right\|_{\boldsymbol{H}(\operatorname{div}, \Omega)}=\left\|\mathbf{W}_{l}\right\|_{L_{2}(\Omega)} \leq\left\|\mathbf{W}_{l}-\mathbf{W}\right\|_{L_{2}(\Omega)}+\|\mathbf{W}\|_{L_{2}(\Omega)}
$$

Lemma 4.5. There exist operators $\mathrm{E}_{l}^{1}: \mathcal{R} \mathcal{T}_{0}\left(\Gamma_{l}\right) \rightarrow \mathcal{N D}_{1}\left(\Omega_{l}\right), \widetilde{\mathrm{E}}_{l}^{1}: \widetilde{\mathcal{R T}}_{0}\left(\Gamma_{l}^{+}\right) \rightarrow$ $\widehat{\mathcal{N D}}_{1}\left(\Omega_{l}\right)$, and $\widehat{\mathrm{E}}_{l}^{1}: \mathcal{R} \mathcal{T}_{0}\left(\Gamma_{l}^{+}\right) \rightarrow \mathcal{N} \mathcal{D}_{1}\left(\Omega_{l}\right)$ that are extensions for all $l \in \mathbb{N}_{0}$ :

$$
\begin{array}{ll}
\mathrm{T}_{t} \mathrm{E}_{l}^{1} \mathbf{v}_{l}=\mathbf{v}_{l}, & \text { on } \Gamma, \quad \forall \mathbf{v}_{l} \in \mathcal{R} \mathcal{T}_{0}\left(\Gamma_{l}\right), \\
\mathrm{T}_{t} \widetilde{\mathrm{E}}_{l}^{1} \widetilde{\mathbf{v}}_{l}=\widetilde{\mathbf{v}}_{l}, & \text { on } \Gamma, \quad \forall \widetilde{\mathbf{v}}_{l} \in \widetilde{\mathcal{R}}_{0}\left(\Gamma_{l}^{+}\right), \\
\mathrm{T}_{t} \widehat{\mathrm{E}}_{l}^{1} \widehat{\mathbf{v}}_{l}=\widehat{\mathbf{v}}_{l}, & \text { on } \Gamma^{+}, \quad \forall \widehat{\mathbf{v}}_{l} \in \mathcal{R} \mathcal{T}_{0}\left(\Gamma_{l}^{+}\right),
\end{array}
$$

and that enjoy the l-uniform stability properties

$$
\begin{aligned}
\left\|\mathrm{E}_{l}^{1} \mathbf{v}_{l}\right\|_{\boldsymbol{H}(\mathbf{c u r l}, \Omega)} \lesssim\left\|\mathbf{v}_{l}\right\|_{\boldsymbol{H}^{-\frac{1}{2}}\left(\operatorname{div}_{\Gamma}, \Gamma\right)}, \quad \forall \mathbf{v}_{l} \in \mathcal{R} \mathcal{T}_{0}\left(\Gamma_{l}\right), \forall l \in \mathbb{N}_{0}, \\
\left\|\widetilde{\mathrm{E}}_{l}^{1} \widetilde{\mathbf{v}}_{l}\right\|_{\boldsymbol{H}(\operatorname{curl}, \Omega)} \lesssim\left\|\widetilde{\mathbf{v}}_{l}\right\|_{\boldsymbol{H}^{-\frac{1}{2}}\left(\operatorname{div}_{\Gamma}, \Gamma\right)}, \quad \forall \widetilde{\mathbf{v}}_{l} \in \widetilde{\mathcal{R} \mathcal{T}_{0}}\left(\Gamma_{l}^{+}\right), \forall l \in \mathbb{N}_{0}, \\
\left\|\widehat{\mathrm{E}}_{l}^{1} \widehat{\mathbf{v}}_{l}\right\|_{\boldsymbol{H}(\operatorname{curl}, \Omega)} \lesssim\left\|\widehat{\mathbf{v}}_{l}\right\|_{\boldsymbol{H}^{-\frac{1}{2}}\left(\operatorname{div}_{\Gamma}, \Gamma+\right)}, \quad \forall \widehat{\mathbf{v}}_{l} \in \boldsymbol{\mathcal { R }}_{0}\left(\Gamma_{l}^{+}\right), \forall l \in \mathbb{N}_{0} .
\end{aligned}
$$

Proof. The assertion for the operators $\mathrm{E}_{l}^{1}$ and $\widetilde{\mathrm{E}}_{l}^{1}$ is covered by [29, Lemma 5.5]. Mere alterations of the proof are sufficient to deal with $\widehat{E}_{l}^{1}$, but we elaborate them for the sake of completeness.

Given $\widehat{\mathbf{v}}_{l} \in \mathcal{R} \mathcal{T}_{0}\left(\Gamma_{l}^{+}\right)$, we know $\operatorname{div}_{\Gamma} \widehat{\mathbf{v}}_{l} \in \mathcal{Q}_{0}\left(\Gamma_{l}^{+}\right)$, which makes it possible to invoke the discrete extension $\widehat{\mathrm{E}}_{l}^{2}$ from Lemma 4.4 on $\operatorname{div}_{\Gamma} \widehat{\mathbf{v}}_{l}$. We have assumed 
trivial topology of $\Omega$. Thus, as $\widehat{\mathrm{E}}_{l}^{2} \operatorname{div}_{\Gamma} \widehat{\mathbf{v}}_{l} \in \boldsymbol{\mathcal { R }} \boldsymbol{\mathcal { T }}_{0}\left(\Omega_{l}\right) \cap \boldsymbol{H}(\operatorname{div} 0, \Omega)$ we conclude from [26, Thm. 3.1] and [26, Cor. 4.4] that there is a stable discrete vector potential:

$$
\exists \mathbf{U}_{l} \in \mathcal{N D}_{1}\left(\Omega_{l}\right): \quad\left\{\begin{array}{c}
\operatorname{curl} \mathbf{U}_{l}=\widehat{\mathrm{E}}_{l}^{2} \operatorname{div}_{\Gamma} \widehat{\mathbf{v}}_{l}, \\
\left\|\mathbf{U}_{l}\right\|_{\boldsymbol{H}(\operatorname{curl}, \Omega)} \lesssim\left\|\widehat{\mathrm{E}}_{l}^{2} \operatorname{div}_{\Gamma} \widehat{\mathbf{v}}_{l}\right\|_{L_{2}(\Omega)}
\end{array}\right.
$$

Next, we exploit another commuting diagram property analogous to (4.10):

$$
\operatorname{div}_{\Gamma} \circ \mathrm{T}_{\boldsymbol{t}}=\mathrm{T}_{\boldsymbol{n}} \circ \mathbf{c u r l}
$$

which naturally holds on $\mathcal{N D}_{1}\left(\Omega_{l}\right)$. It allows us to conclude that

$$
\begin{aligned}
\operatorname{div}_{\Gamma}\left(\widehat{\mathbf{v}}_{l}-\mathrm{T}_{\boldsymbol{t}} \mathbf{U}_{l}\right) & =\operatorname{div}_{\Gamma} \mathbf{v}_{l}-\mathrm{T}_{\boldsymbol{n}} \operatorname{curl} \mathbf{U}_{l} \\
& =\operatorname{div}_{\Gamma} \widehat{\mathbf{v}}_{l}-\mathrm{T}_{\boldsymbol{n}} \widehat{\mathrm{E}}_{l}^{2} \operatorname{div}_{\Gamma} \widehat{\mathbf{v}}_{l} \stackrel{\operatorname{Lemma}}{=} 0 \text { on } \Gamma^{+} .
\end{aligned}
$$

As a consequence, as $\Gamma^{+}$is simply connected, we find $\widehat{p}_{l} \in \mathcal{S}_{1}\left(\Gamma_{l}^{+}\right)$such that

$$
\operatorname{curl}_{\Gamma} \widehat{p}_{l}=\widehat{\mathbf{v}}_{l}-\mathrm{T}_{t} \mathrm{U}_{l} .
$$

According to Proposition 6.2 of [12], $\operatorname{curl}_{\Gamma^{+}}: H^{\frac{1}{2}}\left(\Gamma^{+}\right) \rightarrow \boldsymbol{H}^{-\frac{1}{2}}\left(\operatorname{div}_{\Gamma}, \Gamma^{+}\right)$is continuous and injective with closed range, which implies

$$
\begin{aligned}
& \left\|\widehat{p}_{l}\right\|_{H^{\frac{1}{2}}\left(\Gamma^{+}\right)} \lesssim\left\|\operatorname{curl}_{\Gamma} \widehat{p}_{l}\right\|_{\boldsymbol{H}_{\|}^{-\frac{1}{2}}\left(\Gamma^{+}\right)}=\left\|\widehat{\mathbf{v}}_{l}-\mathrm{T}_{\boldsymbol{t}} \mathbf{U}_{l}\right\|_{\boldsymbol{H}_{\|}^{-\frac{1}{2}}\left(\Gamma^{+}\right)} \\
& \lesssim\left(\left\|\mathbf{v}_{l}\right\|_{\boldsymbol{H}_{\|}^{-\frac{1}{2}}\left(\Gamma^{+}\right)}+\left\|\mathbf{U}_{l}\right\|_{\boldsymbol{H}(\operatorname{curl}, \Omega)}\right) \stackrel{\text { 年.14 }}{\lesssim}\left\|\mathbf{v}_{l}\right\|_{\boldsymbol{H}^{-\frac{1}{2}}\left(\operatorname{div}_{\Gamma}, \Gamma^{+}\right)} .
\end{aligned}
$$

Now, we consider an $H^{1}(\Omega)$-extension of $\widehat{p}_{l}$ defined as the solution $V \in H^{1}(\Omega)$ of the auxiliary boundary value problem

$$
\left\{\begin{aligned}
-\Delta V+V & =0, & & \text { in } \quad \Omega, \\
\mathrm{T}_{\boldsymbol{x}} V & =\widehat{p}_{l}, & & \text { on } \Gamma^{+}, \\
\frac{\partial V}{\partial \boldsymbol{n}} & =0, & & \text { on } \quad \Gamma \backslash \Gamma^{+}
\end{aligned}\right.
$$

which satisfies the regularity estimate

$$
\|V\|_{H^{1}(\Omega)} \leq C\left\|\widehat{p}_{l}\right\|_{H^{\frac{1}{2}}\left(\Gamma^{+}\right)},
$$

where the constant $C>0$ depends only on the domain $\Omega$ and $\Gamma^{+}$. Use this boundary value problem to define $V_{l}:=\mathrm{Q}_{l} V$, where $\mathrm{Q}_{l}: H^{1}(\Omega) \rightarrow \mathcal{S}_{1}\left(\Omega_{l}\right)$ stands for the classical Scott-Zhang quasi-interpolation operator, which is continuous and preserves boundary values in $\mathcal{S}_{1}\left(\Gamma_{l}^{+}\right)$; see [43].

Eventually, we can define $\widehat{\mathrm{E}}_{l}^{2} \widehat{\mathbf{v}}_{l}:=\mathbf{U}_{l}+\operatorname{grad} V_{l}$, which gives the desired stable discrete extension operator, because

$$
\begin{aligned}
\left\|\widehat{\mathrm{E}}_{l}^{2} \widehat{\mathbf{v}}_{l}\right\|_{\boldsymbol{H}(\mathbf{c u r l}, \Omega)} & \lesssim\left\|\mathbf{U}_{l}\right\|_{\boldsymbol{H}(\mathbf{c u r l}, \Omega)}+\left\|V_{l}\right\|_{H^{1}(\Omega)} \\
& \stackrel{(4.14)}{\lesssim}\left\|\operatorname{div}_{\Gamma} \widehat{\mathbf{v}}_{l}\right\|_{H^{-\frac{1}{2}\left(\Gamma^{+}\right)}}+\left\|\widehat{p}_{l}\right\|_{H^{\frac{1}{2}\left(\Gamma^{+}\right)}} \lesssim\left\|\widehat{\mathbf{v}}_{l}\right\|_{\boldsymbol{H}^{-\frac{1}{2}}\left(\operatorname{div}_{\Gamma}, \Gamma^{+}\right)}
\end{aligned}
$$

and

$$
\mathrm{T}_{\boldsymbol{t}} \widehat{\mathrm{E}}_{l}^{1} \widehat{\mathbf{v}}_{l}=\mathrm{T}_{\boldsymbol{t}} \mathbf{U}_{l}+\mathrm{T}_{\boldsymbol{t}}\left(\operatorname{grad} V_{l}\right) \stackrel{(4.19)}{=} \mathrm{T}_{\boldsymbol{t}} \mathbf{U}_{l}+\operatorname{curl}_{\Gamma}\left(\mathrm{T}_{\boldsymbol{x}} V_{l}\right)=\mathrm{T}_{\boldsymbol{t}} \mathrm{U}_{l}+\widehat{p}_{l} \quad \text { on } \Gamma^{+},
$$

where we relied on a third commuting diagram property for traces

$$
\mathrm{T}_{t} \circ \operatorname{grad}=\operatorname{curl}_{\Gamma} \circ \mathrm{T}_{\boldsymbol{x}} .
$$




\section{Equivalent MultileVEL NORMS}

Armed with the extension operators of Section 4 we can resort to the abstract procedure of [29, Sect. 2] to transfer stable multilevel splittings from the domain to the boundary. Its concrete application was already demonstrated in the proof of Theorem 3.4 and we essentially follow these lines.

The theory of (local) multilevel preconditioning for edge elements developed in [25, 27, 31] and [48, Sect. 5] suggests the following counterparts of the multilevel norms (3.1)-(3.2) for $\boldsymbol{H}(\mathbf{c u r l}, \Omega)$-conforming finite elements:

$$
\left\|\mathbf{V}_{L}\right\|_{\mathcal{N D}_{1}\left(\Omega_{L}\right)}^{2}:=\inf \left\{\begin{array}{l}
\sum_{l=0}^{L} h_{l}^{-2}\left(\left\|\mathbf{W}_{l}\right\|_{L_{2}(\Omega)}^{2}+\left\|U_{l}\right\|_{L_{2}(\Omega)}^{2}\right), \mathbf{W}_{l} \in \mathcal{N D}_{1}\left(\Omega_{l}\right), \\
U_{l} \in \mathcal{S}_{1}\left(\Omega_{l}\right), \sum_{l=0}^{L} \mathbf{W}_{l}+\operatorname{grad} U_{l}=\mathbf{V}_{L}
\end{array}\right\},
$$

for $\mathbf{V}_{L} \in \mathcal{N} \mathcal{D}_{1}\left(\Omega_{L}\right)$, and

$$
\left\|\widetilde{\mathbf{V}}_{L}\right\|_{\widetilde{\mathcal{N}}_{1}\left(\Omega_{L}\right)}^{2}:=\inf \left\{\begin{array}{l}
\sum_{l=0}^{L} h_{l}^{-2}\left(\left\|\widetilde{\mathbf{W}}_{l}\right\|_{L_{2}(\Omega)}^{2}+\left\|\widetilde{U}_{l}\right\|_{L_{2}(\Omega)}^{2}\right), \widetilde{\mathbf{W}}_{l} \in{\widetilde{\mathcal{N} \mathcal{D}_{1}}}_{1}\left(\Omega_{l}\right), \\
\widetilde{U}_{l} \in \widetilde{\mathcal{S}}_{1}\left(\Omega_{l}\right), \sum_{l=0}^{L} \widetilde{\mathbf{W}}_{l}+\operatorname{grad} \widetilde{U}_{l}=\widetilde{\mathbf{V}}_{L}
\end{array}\right\},
$$

for $\widetilde{\mathbf{V}}_{L} \in \widetilde{\mathcal{N D}}_{1}\left(\Omega_{L}\right)$. The underlying splittings are stable in the sense of norm equivalence; cf. Theorem 3.3 .

Theorem 5.1. The following norms are equivalent:

$$
\begin{aligned}
&\left\|\mathbf{V}_{L}\right\|_{\mathcal{N D}_{1}\left(\Omega_{L}\right)} \approx\left\|\mathbf{V}_{L}\right\|_{H(\operatorname{curl}, \Omega)}, \forall \mathbf{V}_{L} \in \mathcal{N} \mathcal{D}_{1}\left(\Omega_{L}\right), \\
&\left\|\widetilde{\mathbf{V}}_{L}\right\|_{\widetilde{\mathcal{N D}}_{1}\left(\Omega_{L}\right)} \approx\left\|\widetilde{\mathbf{V}}_{L}\right\|_{\boldsymbol{H}(\operatorname{curl}, \Omega)}, \quad \forall \widetilde{\mathbf{V}}_{L} \in \widetilde{\mathcal{N D}}_{1}\left(\Omega_{L}\right) .
\end{aligned}
$$

Proof. Results that can be combined into a proof of this theorem can be found in [14,25, 27,48; in particular, see [31, Lemma 5.2 \& Thm. 4.2].

Similar norm equivalences also hold for the edge boundary element spaces $\boldsymbol{R} \mathcal{T}_{0}\left(\Gamma_{L}\right)$ relying on the multilevel norms

$$
\left\|\mathbf{v}_{L}\right\|_{\mathcal{R}_{0}\left(\Gamma_{L}\right)}^{2}:=\inf \left\{\begin{array}{l}
\sum_{l=0}^{L} h_{l}^{-1}\left(\left\|\mathbf{w}_{l}\right\|_{L_{2}(\Gamma)}^{2}+\left\|q_{l}\right\|_{L_{2}(\Gamma)}^{2}\right), \mathbf{w}_{l} \in \mathcal{R} \mathcal{T}_{0}\left(\Gamma_{l}\right), \\
q_{l} \in \mathcal{S}_{1}\left(\Gamma_{l}\right), \sum_{l=0}^{L}\left(\mathbf{w}_{l}+\operatorname{curl}_{\Gamma} q_{l}\right)=\mathbf{v}_{L}
\end{array}\right\},
$$


for $\mathbf{v}_{L} \in \mathcal{R} \mathcal{T}_{0}\left(\Gamma_{L}\right)$, and

$$
\left\|\widetilde{\mathbf{v}}_{L}\right\|_{\widetilde{\mathcal{R T}}_{0}\left(\Gamma_{L}^{+}\right)}^{2}:=\inf \left\{\begin{array}{l}
\sum_{l=0}^{L} h_{l}^{-1}\left(\left\|\widetilde{\mathbf{w}}_{l}\right\|_{L_{2}(\Gamma)}^{2}+\left\|\widetilde{q}_{l}\right\|_{L_{2}(\Gamma)}^{2}\right) ; \widetilde{\mathbf{w}}_{l} \in \widetilde{\mathcal{R}}_{0}\left(\Gamma_{l}^{+}\right), \\
\widetilde{q}_{l} \in \widetilde{\mathcal{S}}_{1}\left(\Gamma_{l}^{+}\right), \quad \sum_{l=0}^{L}\left(\widetilde{\mathbf{w}}_{l}+\operatorname{curl}_{\Gamma} \widetilde{q}_{l}\right)=\widetilde{\mathbf{v}}_{L}
\end{array}\right\},
$$

for $\widetilde{\mathbf{v}}_{L} \in \widetilde{\mathcal{R} \mathcal{T}}_{0}\left(\Gamma_{L}^{+}\right)$.

The multilevel norms play roles similar to those of (3.1) and (3.2) in Section 3 . Accordingly, we obtain the following norm equivalences.

Theorem 5.2. The following uniform norm equivalences hold for surface edge elements:

$$
\begin{array}{cl}
\left\|\mathbf{v}_{L} \mid\right\|_{\mathcal{R}_{0}\left(\Gamma_{L}\right)} \approx\left\|\mathbf{v}_{L}\right\|_{\boldsymbol{H}^{-\frac{1}{2}}\left(\operatorname{div}_{\Gamma}, \Gamma\right)}, \quad \forall \mathbf{v}_{L} \in \boldsymbol{\mathcal { R }}_{0}\left(\Gamma_{L}\right), \\
\left\|\widetilde{\mathbf{v}}_{L}\right\|_{\widetilde{\mathcal{R}}_{0}\left(\Gamma_{L}^{+}\right)} \approx\left\|\widetilde{\mathbf{v}}_{L}\right\|_{\widetilde{\boldsymbol{H}}^{-\frac{1}{2}}\left(\operatorname{div}_{\Gamma}, \Gamma^{+}\right)}, \quad \forall \widetilde{\mathbf{v}}_{L} \in{\widetilde{\mathcal{R} \mathcal{T}_{0}}}_{0}\left(\Gamma_{L}^{+}\right) .
\end{array}
$$

Proof. We use the extension operators provided by Lemmas 4.1, 4.5, and 3.2, 3.1 together with Theorem 5.1 in the very same fashion as in the proof of Theorem 3.4 . see also [29, Sect. 6].

We only give a proof of (5.6); (5.5) can be inferred by a similar argument.

(1 In order to prove the "¿" estimate, pick an arbitrary $\widetilde{\mathbf{v}}_{L} \in \widetilde{\mathcal{R T}}_{0}\left(\Gamma_{L}^{+}\right)$. Then, by Lemma 4.5. we know that $\widetilde{\mathrm{E}}_{L}^{1} \widetilde{\mathbf{v}}_{L} \in \widehat{\mathcal{N D}}_{1}\left(\Omega_{L}\right)$ satisfies

$$
\mathrm{T}_{\boldsymbol{t}} \widetilde{\mathrm{E}}_{L}^{1} \widetilde{\mathbf{v}}_{L}=\widetilde{\mathbf{v}}_{L}, \quad\left\|\widetilde{\mathrm{E}}_{L}^{1} \widetilde{\mathbf{v}}_{L}\right\|_{\boldsymbol{H}(\operatorname{curl}, \Omega)} \lesssim\left\|\widetilde{\mathbf{v}}_{L}\right\|_{\boldsymbol{H}^{-\frac{1}{2}}\left(\operatorname{div}_{\Gamma}, \Gamma\right)} \approx\left\|\widetilde{\mathbf{v}}_{L}\right\|_{\widetilde{\boldsymbol{H}}^{-\frac{1}{2}}\left(\operatorname{div}_{\Gamma}, \Gamma^{+}\right)},
$$

which, together with the trace inequality,

$$
\left\|\widetilde{\mathbf{v}}_{L}\right\|_{\widetilde{\boldsymbol{H}}^{-\frac{1}{2}}\left(\operatorname{div}_{\Gamma}, \Gamma^{+}\right)} \approx\left\|\widetilde{\mathbf{v}}_{L}\right\|_{\boldsymbol{H}^{-\frac{1}{2}}\left(\operatorname{div}_{\Gamma}, \Gamma\right)} \lesssim\left\|\widetilde{\mathrm{E}}_{L}^{1} \widetilde{\mathbf{v}}_{L}\right\|_{\boldsymbol{H}(\mathbf{c u r l}, \Omega)}
$$

and Theorem 5.1. implies that

$$
\left\|\widetilde{\mathbf{v}}_{L}\right\|_{\widetilde{\boldsymbol{H}}^{-\frac{1}{2}}\left(\operatorname{div}_{\Gamma}, \Gamma^{+}\right)} \approx\left\|\widetilde{\mathrm{E}}_{L}^{1} \widetilde{\mathbf{v}}_{L}\right\|_{\boldsymbol{H}(\operatorname{curl}, \Omega)} \approx\left\|\widetilde{\mathrm{E}}_{L}^{1} \widetilde{\mathbf{v}}_{L}\right\|_{\widetilde{\mathcal{N D}}_{1}\left(\Omega_{L}\right)} .
$$

Thus, we need only prove

$$
\left\|\widetilde{\mathbf{v}}_{L} \mid\right\|_{\widetilde{\mathcal{R T}}_{0}\left(\Gamma_{L}^{+}\right)} \lesssim\left\|\widetilde{\mathrm{E}}_{L}^{1} \widetilde{\mathbf{v}}_{L}\right\|_{\widetilde{\mathcal{N D}}_{1}\left(\Omega_{L}\right)}
$$

For any splitting of $\widetilde{\mathrm{E}}_{L}^{1} \widetilde{\mathbf{v}}_{L}$ of the form

$$
\sum_{l=0}^{L}\left(\widetilde{\mathbf{W}}_{l}+\operatorname{grad} \widetilde{U}_{l}\right)=\widetilde{\mathrm{E}}_{L}^{1} \widetilde{\mathbf{v}}_{L},
$$

we can define

$$
\widetilde{\mathbf{w}}_{l}:=\mathrm{T}_{\boldsymbol{t}} \widetilde{\mathbf{W}}_{l} \in \widetilde{\mathcal{R}}_{0}\left(\Gamma_{l}^{+}\right), \quad \widetilde{q}_{l}:=\mathrm{T}_{\boldsymbol{x}} \widetilde{U}_{l} \in \widetilde{\mathcal{S}}_{1}\left(\Gamma_{l}^{+}\right),
$$

which, by the commuting diagram property (4.19), means $\operatorname{curl}_{\Gamma} \widetilde{q}_{l}=\mathrm{T}_{t} \operatorname{grad} \widetilde{U}_{l}$ so that we have

$$
\sum_{l=0}^{L}\left(\widetilde{\mathbf{w}}_{l}+\operatorname{curl}_{\Gamma} \widetilde{q}_{l}\right)=\mathrm{T}_{t} \widetilde{\mathrm{E}}_{L}^{1} \widetilde{\mathbf{v}}_{L}=\widetilde{\mathbf{v}}_{L}
$$


By a simple scaling argument we verify that

$$
\left\|\widetilde{\mathbf{w}}_{l}\right\|_{L_{2}(\Gamma)}^{2}+\left\|\widetilde{q}_{l}\right\|_{L_{2}(\Gamma)}^{2} \lesssim h_{l}^{-1}\left(\left\|\widetilde{\mathbf{W}}_{l}\right\|_{L_{2}(\Omega)}+\left\|\widetilde{U}_{l}\right\|_{L_{2}(\Omega)}\right),
$$

which implies the "¿" estimate in (5.10).

(2) We next establish the "邓" estimate. For any splitting of $\widetilde{\mathbf{v}}_{L}$ with

$$
\sum_{l=0}^{L} \widetilde{\mathbf{w}}_{l}+\operatorname{curl}_{\Gamma} \widetilde{q}_{l}=\widetilde{\mathbf{v}}_{L}, \quad \widetilde{\mathbf{w}}_{l} \in \widetilde{\mathcal{R}}_{0}\left(\Gamma_{l}^{+}\right), \quad \widetilde{q}_{l} \in \widetilde{\mathcal{S}}_{1}\left(\Gamma_{l}^{+}\right),
$$

we apply the results of Lemmas 3.2 and 4.1 by setting

$$
\widetilde{\mathbf{W}}_{l}:=\widetilde{\mathbf{N}}_{l}^{1} \widetilde{\mathbf{w}}_{l} \in{\widetilde{\mathcal{N} \mathcal{D}_{1}}}_{1}\left(\Omega_{l}\right), \quad \widetilde{U}_{l}:=\widetilde{\mathbf{N}}_{l}^{0} \widetilde{q}_{l} \in \widetilde{\mathcal{S}}_{1}\left(\Omega_{l}\right),
$$

with the obvious property

$$
\mathrm{T}_{t}\left(\sum_{l=0}^{L} \widetilde{\mathbf{W}}_{l}+\operatorname{grad} \widetilde{U}_{l}\right)=\widetilde{\mathbf{v}}_{L} .
$$

Then, by the continuity of the trace operator $T_{t}$, we can derive

$$
\begin{aligned}
& \sum_{l=0}^{L} h_{l}^{-1}\left(\left\|\widetilde{\mathbf{w}}_{l}\right\|_{L_{2}(\Gamma)}^{2}+\left\|\widetilde{q}_{l}\right\|_{L_{2}(\Gamma)}^{2}\right) \\
& \underset{\text { Lemmas }}{2.2} \sum_{l=0}^{L .1} h_{l}^{-2}\left(\left\|\widetilde{\mathrm{N}}_{l}^{1} \widetilde{\mathbf{w}}_{l}\right\|_{L_{2}(\Omega)}^{2}+\left\|\widetilde{\mathrm{N}}_{l}^{0} \widetilde{q}_{l}\right\|_{L_{2}(\Omega)}^{2}\right) \\
& \stackrel{\text { infimum }}{\gtrsim}\left\|\sum_{l=0}^{L}\left(\widetilde{\mathbf{W}}_{l}+\operatorname{grad} \widetilde{U}_{l}\right)\right\|_{\widetilde{\mathcal{N D}}_{1}\left(\Omega_{L}\right)}^{2} \\
& \stackrel{\text { Theorem }[5.1}{\gtrsim}\left\|\sum_{l=0}^{L}\left(\widetilde{\mathbf{W}}_{l}+\operatorname{grad} \widetilde{U}_{l}\right)\right\|_{\boldsymbol{H}(\mathbf{c u r l}, \Omega)}^{2} \\
& \gtrsim \quad\left\|\mathrm{T}_{\boldsymbol{t}}\left(\sum_{l=0}^{L}\left(\widetilde{\mathbf{W}}_{l}+\operatorname{grad} \widetilde{U}_{l}\right)\right)\right\|_{\boldsymbol{H}^{-\frac{1}{2}}\left(\operatorname{div}_{\Gamma}, \Gamma\right)} \\
& \gtrsim \quad\left\|\widetilde{\mathbf{v}}_{L}\right\|_{\boldsymbol{H}^{-\frac{1}{2}}\left(\operatorname{div}_{\Gamma}, \Gamma\right)}^{2} \approx\left\|\widetilde{\mathbf{v}}_{L}\right\|_{\widetilde{\boldsymbol{H}}^{-\frac{1}{2}}\left(\operatorname{div}_{\Gamma}, \Gamma^{+}\right)}^{2} .
\end{aligned}
$$

proving the desired inequality.

In Section 3 we relied on a third multilevel norm (3.9) to deal with localized trace norms. Here, we follow the same idea and consider

$$
\left\|\mathbf{v}_{L}\right\|_{\mathcal{R}_{\mathcal{T}_{0}\left(\Gamma_{L}^{+}\right)}^{2}}^{2}=\inf \left\{\begin{array}{l}
\sum_{l=0}^{L} h_{l}^{-1}\left(\left\|\mathbf{w}_{l}\right\|_{L_{2}(\Gamma)}^{2}+\left\|q_{l}\right\|_{L_{2}(\Gamma)}^{2}\right), \mathbf{w}_{l} \in \mathcal{R} \mathcal{T}_{0}\left(\Gamma_{l}\right), \\
q_{l} \in \mathcal{S}_{1}\left(\Gamma_{l}\right),\left.\left(\sum_{l=0}^{L}\left(\mathbf{w}_{l}+\operatorname{curl}_{\Gamma} q_{l}\right)\right)\right|_{\Gamma^{+}}=\mathbf{v}_{L}
\end{array}\right\} .
$$

Note that the norms for the terms in the splitting are computed on the entire boundary $\Gamma$. Then a counterpart of Lemma 3.5 holds:

Theorem 5.3. On $\mathcal{R} \mathcal{T}_{0}\left(\Gamma_{L}^{+}\right)$the following norms are equivalent:

$$
\left\|\mathbf{v}_{L}\right\|_{\boldsymbol{H}^{-\frac{1}{2}}\left(\operatorname{div}_{\Gamma}, \Gamma^{+}\right)} \approx\left\|\mathbf{v}_{L}\right\|_{\mathcal{R} \mathcal{T}_{0}\left(\Gamma_{L}^{+}\right)}, \quad \forall \mathbf{v}_{L} \in \mathcal{R} \mathcal{T}_{0}\left(\Gamma_{L}^{+}\right) .
$$


Proof. With the extension operators of Lemma 4.5 at our disposal, the proof largely follows that of Lemma 3.5 with a minor change: we have to employ the discrete extension operator $\widehat{E}_{l}^{1}$ from Lemma 4.5] instead of the Scott-Zhang quasi-interpolation operator.

Concerning lowest-order $\boldsymbol{H}(\operatorname{div}, \Omega)$-conforming finite elements (Raviart-Thomas elements, face elements), the theory of (local) multilevel preconditioning was developed in [4,24 and [48, Theorem 5.8]. The results suggest the following definition of multilevel norms in analogy to (5.3) and (5.4):

$$
\left\|\mathbf{X}_{L}\right\|_{\mathcal{R}_{0}\left(\Omega_{L}\right)}^{2}:=\inf \left\{\begin{array}{l}
\sum_{l=0}^{L} h_{l}^{-2}\left(\left\|\mathbf{Y}_{l}\right\|_{L_{2}(\Omega)}^{2}+\left\|\mathbf{Z}_{l}\right\|_{L_{2}(\Omega)}^{2}\right), \mathbf{Y}_{l} \in \mathcal{R} \mathcal{T}_{0}\left(\Omega_{l}\right), \\
\mathbf{Z}_{l} \in \mathcal{N} \mathcal{D}_{1}\left(\Omega_{l}\right), \sum_{l=0}^{L}\left(\mathbf{Y}_{l}+\operatorname{curl} \mathbf{Z}_{l}\right)=\mathbf{X}_{L}
\end{array}\right\},
$$

for $\mathbf{X}_{L} \in \mathcal{R} \mathcal{T}_{0}\left(\Omega_{L}\right)$, and

$$
\left\|\widetilde{\mathbf{X}}_{L}\right\|_{\widetilde{\mathcal{R T}}_{0}\left(\Omega_{L}\right)}^{2}:=\inf \left\{\begin{array}{l}
\sum_{l=0}^{L} h_{l}^{-2}\left(\left\|\widetilde{\mathbf{Y}}_{l}\right\|_{L_{2}(\Omega)}^{2}+\left\|\widetilde{\mathbf{Z}}_{l}\right\|_{L_{2}(\Omega)}^{2}\right), \widetilde{\mathbf{Y}}_{l} \in \widetilde{\mathcal{R}}_{0}\left(\Omega_{l}\right), \\
\widetilde{\mathbf{Z}}_{l} \in \widetilde{\mathcal{N} \mathcal{D}_{1}}\left(\Omega_{l}\right), \sum_{l=0}^{L}\left(\widetilde{\mathbf{Y}}_{l}+\operatorname{curl} \widetilde{\mathbf{Z}}_{l}\right)=\widetilde{\mathbf{X}}_{L}
\end{array}\right\},
$$

for $\widetilde{\mathbf{X}}_{L} \in \widetilde{\mathcal{R T}}_{0}\left(\Omega_{L}\right)$. The underlying splittings are stable in the sense of norm equivalence; cf. Theorem 3.3 .

Theorem 5.4. The following norms are equivalent:

$$
\begin{aligned}
\left\|\mathbf{X}_{L}\right\|_{\mathcal{R T}_{0}\left(\Omega_{L}\right)} \approx\left\|\mathbf{X}_{L}\right\|_{\boldsymbol{H}(\operatorname{div}, \Omega)}, \quad \forall \mathbf{X}_{L} \in \mathcal{R} \mathcal{T}_{0}\left(\Omega_{L}\right), \\
\left\|\widetilde{\mathbf{X}}_{L}\right\|_{\widetilde{\mathcal{R T}}_{0}\left(\Omega_{L}\right)} \approx\left\|\widetilde{\mathbf{X}}_{L}\right\|_{\boldsymbol{H}(\operatorname{div}, \Omega)}, \quad \forall \widetilde{\mathbf{X}}_{L} \in \widetilde{\mathcal{R}}_{0}\left(\Omega_{L}\right) .
\end{aligned}
$$

Proof. A proof of this theorem can be built by combining results provided in $4,5,24]$ and [48, Theorem 5.8].

Norm equivalences as expressed in Theorem 5.2 also hold for spaces of piecewise constant functions on $\Gamma_{L}$. They rely on multilevel norms corresponding to (5.3) and (5.4)

$$
\left\|\varphi_{L}\right\|_{\mathcal{Q}_{0}\left(\Gamma_{L}\right)}^{2}:=\inf \left\{\begin{array}{l}
\sum_{l=0}^{L} h_{l}^{-1}\left(\left\|\eta_{l}\right\|_{L_{2}(\Gamma)}^{2}+\left\|\mathbf{q}_{l}\right\|_{L_{2}(\Gamma)}^{2}\right), \eta_{l} \in \mathcal{Q}_{0}\left(\Gamma_{l}\right), \\
\mathbf{q}_{l} \in \mathcal{R} \mathcal{T}_{0}\left(\Gamma_{l}\right), \sum_{l=0}^{L}\left(\eta_{l}+\operatorname{div}_{\Gamma} \mathbf{q}_{l}\right)=\varphi_{L}
\end{array}\right\}
$$


for $\varphi_{L} \in \mathcal{Q}_{0}\left(\Gamma_{L}\right)$, and

$$
\left\|\widetilde{\varphi}_{L}\right\|_{\widetilde{\mathcal{Q}}_{0}\left(\Gamma_{L}^{+}\right)}^{2}:=\inf \left\{\begin{array}{l}
\sum_{l=0}^{L} h_{l}^{-1}\left(\left\|\widetilde{\eta}_{l}\right\|_{L_{2}(\Gamma)}^{2}+\left\|\widetilde{\mathbf{q}}_{l}\right\|_{L_{2}(\Gamma)}^{2}\right) \widetilde{\eta}_{l} \in \widetilde{\mathcal{Q}}_{0}\left(\Gamma_{l}^{+}\right), \\
\widetilde{\mathbf{q}}_{l} \in \widetilde{\mathcal{R} \mathcal{T}_{0}}\left(\Gamma_{l}^{+}\right), \sum_{l=0}^{L}\left(\widetilde{\eta}_{l}+\operatorname{div}_{\Gamma} \widetilde{\mathbf{q}}_{l}\right)=\widetilde{\varphi}_{L}
\end{array}\right\},
$$

for $\widetilde{\varphi}_{L} \in \widetilde{\mathcal{Q}}_{0}\left(\Gamma_{L}^{+}\right)$.

Theorem 5.5. The following L-uniform norm equivalences hold:

$$
\begin{gathered}
\left\|\mid \varphi_{L}\right\|_{\mathcal{Q}_{0}\left(\Gamma_{L}\right)} \approx\left\|\varphi_{L}\right\|_{H^{-\frac{1}{2}}(\Gamma)}, \quad \forall \varphi_{L} \in \mathcal{Q}_{0}\left(\Gamma_{L}\right), \\
\left\|\widetilde{\varphi}_{L}\right\|_{\widetilde{\mathcal{Q}}_{0}\left(\Gamma_{L}^{+}\right)} \approx\left\|\widetilde{\varphi}_{L}\right\|_{\widetilde{H}^{-\frac{1}{2}}\left(\Gamma^{+}\right)}, \quad \forall \widetilde{\varphi}_{L} \in \widetilde{\mathcal{Q}}_{0}\left(\Gamma_{L}^{+}\right) .
\end{gathered}
$$

Proof. The proof is very similar to that of Theorem 5.2 and we merely outline minor modifications.

In order to prove the "¿" estimate of (5.18), by Lemma 4.4, the trace inequality and Theorem 5.4 we have to establish

$$
\left\|\widetilde{\varphi}_{L}\right\|_{\widetilde{\mathcal{Q}}_{0}\left(\Gamma_{L}^{+}\right)} \lesssim\left\|\mid \widetilde{\mathrm{E}}_{L}^{2} \widetilde{\varphi}_{L}\right\|_{\widetilde{\mathcal{R T}}_{0}\left(\Omega_{L}\right)},
$$

which can be proved as above in the first part of the proof of Theorem 5.2 after we define

$$
\widetilde{\eta}_{l}=\mathrm{T}_{\boldsymbol{n}} \widetilde{\mathbf{Y}}_{l} \in \widetilde{\mathcal{Q}}_{0}\left(\Gamma_{l}^{+}\right), \quad \widetilde{\mathbf{q}}_{l}:=\mathrm{T}_{t} \widetilde{\mathbf{Z}}_{l} \in \widetilde{\mathcal{R T}}_{0}\left(\Gamma_{l}^{+}\right)
$$

where $\widetilde{\mathrm{E}}_{L}^{2} \widetilde{\varphi}_{L}=\sum_{l=0}^{L}\left(\widetilde{\mathbf{Y}}_{l}+\operatorname{curl} \widetilde{\mathbf{Z}}_{l}\right)$ is a stable multilevel splitting according to Theorem 5.4.

To show the estimate " $\gtrsim$ " of $(\underline{5.18}$ ) we again apply the trivial local extension operators of Lemma 4.2 to the terms of a multilevel splitting of $\widetilde{\varphi}_{L}$. Then, in analogy to the second part of the proof of Theorem 5.2, we use the continuity of the trace operator and the norm equivalence from Theorem 5.4 .

The following theorem gives a multilevel characterization of the localized $H^{-\frac{1}{2}}\left(\Gamma^{+}\right)$-trace norm of piecewise constant functions on $\Gamma^{+}$.

Theorem 5.6. On $\mathcal{Q}_{0}\left(\Gamma_{L}^{+}\right)$the following norms are equivalent:

$$
\left\|\varphi_{L}\right\|_{H^{-\frac{1}{2}}\left(\Gamma^{+}\right)} \approx\left\|\varphi_{L}\right\|_{\mathcal{Q}_{0}\left(\Gamma_{L}^{+}\right)}, \quad \forall \varphi_{L} \in \mathcal{Q}_{0}\left(\Gamma_{L}^{+}\right)
$$

where the multilevel splittings norm is defined by

$$
\left\|\varphi_{L}\right\|_{\mathcal{Q}_{0}\left(\Gamma_{L}^{+}\right)}^{2}:=\inf \left\{\begin{array}{l}
\sum_{l=0}^{L} h_{l}^{-1}\left(\left\|\eta_{l}\right\|_{L_{2}(\Gamma)}^{2}+\left\|\mathbf{q}_{l}\right\|_{L_{2}(\Gamma)}^{2}\right), \eta_{l} \in \mathcal{Q}_{0}\left(\Gamma_{l}\right), \\
\mathbf{q}_{l} \in \mathcal{R} \mathcal{T}_{0}\left(\Gamma_{l}\right),\left.\left(\sum_{l=0}^{L} \eta_{l}+\operatorname{div}_{\Gamma} \mathbf{q}_{l}\right)\right|_{\Gamma^{+}}=\varphi_{L}
\end{array}\right\} .
$$

Proof. We follow exactly the reasoning of the proof of Theorem 5.3 this time using the discrete extension operator $\widehat{\mathrm{E}}_{l}^{2}$ provided by Lemma 4.4. 


\section{Proof: CUt-off inequalities for $\boldsymbol{H}^{-\frac{1}{2}}\left(\operatorname{div}_{\Gamma}, \Gamma\right)-$ AND $H^{-\frac{1}{2}}(\Gamma)$-CONFORMING BEM SPACES}

Finally, we are in position to provide proofs of Theorems 2.1 and 2.2 . In fact, a substantial portion of the work was already done in the two previous sections and now we have to adapt the arguments from the proof of Theorem 3.6. At one step, additional difficulties will crop up, and to deal with them we have to resort to the spaces $\mathcal{S}_{1}\left(\partial \Gamma_{l}^{+}\right), l=0, \ldots, L$, of piecewise linear continuous functions on the curve $\partial \Gamma^{+}$with respect to the mesh $\partial \Gamma_{l}^{+}:=\left.\Gamma_{l}^{+}\right|_{\partial \Gamma^{+}}$. These are simple $C^{0}$-finite element spaces on the one-dimensional domain $\partial \Gamma^{+}$. These spaces come equipped with the standard nodal interpolation operators $\check{\Pi}_{l}$ that amount to piecewise linear interpolation in one dimension.

Differences of linear interpolants define the hierarchical decomposition of $\check{v}_{L} \in$ $\mathcal{S}_{1}\left(\partial \Gamma_{L}^{+}\right)$. It enjoys an elementary but interesting orthogonality property with respect to the $H^{1}\left(\partial \Gamma^{+}\right)$-semi-norm:

$$
\left\|\operatorname{grad}_{\partial \Gamma^{+}} \check{v}_{L}\right\|_{L_{2}\left(\partial \Gamma^{+}\right)}^{2}=\sum_{l=0}^{L}\left\|\operatorname{grad}_{\partial \Gamma^{+}}\left(\check{\Pi}_{l}-\check{\Pi}_{l-1}\right) \check{v}_{L}\right\|_{L_{2}\left(\partial \Gamma^{+}\right)}^{2},
$$

where $\operatorname{grad}_{\partial \Gamma^{+}}$is the gradient along the curve $\partial \Gamma^{+}$, i.e., the tangential derivative along $\partial \Gamma^{+}$, and we set $\check{\Pi}_{-1}:=0$.

Another tool in the proof will be the nodal cut-off for functions in $\mathcal{R} \mathcal{T}_{0}\left(\Gamma_{l}\right)$ defined in analogy to $Z_{l}^{0}$ as

$$
\mathrm{Z}_{l}^{1} \mathbf{v}_{l} \in \mathcal{R} \mathcal{T}_{0}\left(\Gamma_{l}\right): \quad \int_{e}\left(\mathrm{Z}_{l}^{1} \mathbf{v}_{l}\right) \cdot \mathrm{d} \vec{s}= \begin{cases}\int_{e} \mathbf{v}_{l} \cdot \mathrm{d} \vec{s}, & \text { for } e \in \mathcal{E}\left(\Gamma_{l}\right) \cap \Gamma^{+} \\ 0, & \text { for } e \in \mathcal{E}\left(\Gamma_{l}\right), e \not \subset \Gamma^{+}\end{cases}
$$

Proof of Theorem 2.1. (1 The initial reasoning is the same as in the proof of Theorem 3.6 . According to Theorems 5.3 and (5.6) of Theorem 5.2, the assertion of Theorem 2.1 is equivalent to

$$
\left\|\widetilde{\mathbf{v}}_{L}\right\|_{\mathcal{\mathcal { R T }}_{0}\left(\Gamma_{L}^{+}\right)}^{2} \lesssim L^{3}\left\|\widetilde{\mathbf{v}}_{L}\right\|_{\mathcal{R} \mathcal{T}_{0}\left(\Gamma_{L}^{+}\right)}^{2} \quad \forall \widetilde{\mathbf{v}}_{L} \in \widetilde{\mathcal{R T}}_{0}\left(\Gamma_{L}^{+}\right)
$$

compare with (3.11). This means that for any $\widetilde{\mathbf{v}}_{L} \in \widetilde{\mathcal{R}}_{0}\left(\Gamma_{L}^{+}\right) \subset \boldsymbol{\mathcal { R }}_{0}\left(\Gamma_{L}\right)$ and a splitting,

$$
\widetilde{\mathbf{v}}_{L}=\sum_{l=0}^{L}\left(\mathbf{w}_{l}+\operatorname{curl}_{\Gamma} q_{l}\right), \quad \mathbf{w}_{l} \in \mathcal{R} \mathcal{T}_{0}\left(\Gamma_{l}\right), q_{l} \in \mathcal{S}_{1}\left(\Gamma_{l}\right),
$$

we have to find a corresponding multilevel decomposition in functions that vanish outside of $\Gamma^{+}$:

$$
\widetilde{\mathbf{v}}_{L}=\sum_{l=0}^{L}\left(\widetilde{\mathbf{w}}_{l}+\operatorname{curl}_{\Gamma} \widetilde{q}_{l}\right), \quad \widetilde{\mathbf{w}}_{l} \in \widetilde{\mathcal{R}}_{0}\left(\Gamma_{l}^{+}\right), \widetilde{q}_{l} \in \widetilde{\mathcal{S}}_{1}\left(\Gamma_{l}^{+}\right),
$$

and satisfy

$$
\sum_{l=0}^{L} h_{l}^{-1}\left(\left\|\widetilde{\mathbf{w}}_{l}\right\|_{L_{2}(\Gamma)}^{2}+\left\|\widetilde{q}_{l}\right\|_{L_{2}(\Gamma)}^{2}\right) \lesssim L^{3} \sum_{l=0}^{L} h_{l}^{-1}\left(\left\|\mathbf{w}_{l}\right\|_{L_{2}(\Gamma)}^{2}+\left\|q_{l}\right\|_{L_{2}(\Gamma)}^{2}\right),
$$

which corresponds to (3.14) in the proof of Theorem 3.6 . 
(2) Now we deviate from the proof of Theorem 3.6 and take into account that $\widetilde{\mathbf{v}} \in \widetilde{\mathcal{R}}_{0}\left(\Gamma_{L}^{+}\right)$involves

$$
\widetilde{\mathbf{v}}_{L} \cdot \boldsymbol{n}_{\partial \Gamma^{+}}=0 \text { on } \partial \Gamma^{+},
$$

where $\boldsymbol{n}_{\partial \Gamma^{+}}$is the "in-plane exterior unit normal" on $\partial \Gamma^{+}$. However, in general,

$$
\sum_{l=0}^{L} \mathbf{w}_{l} \cdot \boldsymbol{n}_{\partial \Gamma^{+}} \neq 0,\left.\quad \sum_{l=0}^{L} q_{l}\right|_{\partial \Gamma^{+}} \neq 0 .
$$

The following manipulations serve the purpose of altering both of these sums such that they become zero on $\partial \Gamma^{+}$and still sum up to $\widetilde{\mathbf{v}}_{L}$. We rely on an auxiliary hierarchical basis decomposition on $\partial \Gamma^{+}$: it follows from (6.7) that

$$
\sum_{l=0}^{L} \mathbf{w}_{l} \cdot \boldsymbol{n}_{\partial \Gamma^{+}}=-\operatorname{grad}_{\partial \Gamma^{+}} \check{s}_{L}, \quad \check{s}_{L}:=\left.\sum_{l=0}^{L} q_{l}\right|_{\partial \Gamma^{+}} \in \mathcal{S}_{1}\left(\partial \Gamma_{L}^{+}\right) .
$$

Next we consider the hierarchical decomposition of $\check{r}_{L}$,

$$
\check{s}_{L}=\sum_{l=0}^{L} \check{r}_{l}, \quad \check{r}_{l}:=\left(\check{\Pi}_{l}-\check{\Pi}_{l-1}\right) \check{s}_{L} \in \mathcal{S}_{1}\left(\partial \Gamma_{l}^{+}\right),
$$

with $\check{\Pi}_{l}$ the nodal interpolation onto $\mathcal{S}_{1}\left(\partial \Gamma_{l}^{+}\right)$introduced in the beginning of this section.

Modifiers for the $q_{l}$ will be provided by trivial extensions of the $\check{r}_{l}$ to functions $\widehat{r}_{l} \in \mathcal{S}_{1}\left(\Gamma_{l}\right)$ defined by

$$
\widehat{r}_{l}(\boldsymbol{p})= \begin{cases}\check{r}_{l}(\boldsymbol{p}), & \boldsymbol{p} \in \mathcal{V}\left(\Gamma_{l}\right) \cap \partial \Gamma^{+} \\ 0, & \text { otherwise }\end{cases}
$$

which satisfy

$$
\left\|\operatorname{curl}_{\Gamma} \widehat{r}_{l}\right\|_{L_{2}\left(\Gamma^{+}\right)}^{2}=\left\|\operatorname{grad}_{\Gamma} \widehat{r}_{l}\right\|_{L_{2}\left(\Gamma^{+}\right)}^{2} \lesssim h_{l}\left\|\operatorname{grad}_{\partial \Gamma^{+}} \check{r}_{l}\right\|_{L_{2}\left(\partial \Gamma^{+}\right)}^{2} \cdot
$$

Note that

$$
\Upsilon:=\bigcup\left\{T \in \Gamma_{l}^{+}: \bar{T} \cap \Gamma^{+} \neq \emptyset\right\}
$$

is a strip along $\partial \Gamma^{+}$of width $\approx h_{l}$. Thus, noting that $\widehat{r}_{l}$ is a function in $H^{1}(\Upsilon)$ that vanishes on $\partial \Upsilon \backslash \partial \Gamma^{+}$, simple scaling arguments confirm

$$
\left\|\widehat{r}_{l}\right\|_{L_{2}(\Upsilon)}^{2} \lesssim h_{T}^{2}\left\|\operatorname{grad}_{\Gamma} \widehat{r}_{l}\right\|_{L_{2}(\Upsilon)}^{2},
$$

which immediately implies

$$
\left\|\widehat{r}_{l}\right\|_{L_{2}\left(\Gamma^{+}\right)}^{2} \lesssim h_{l}^{2}\left\|\operatorname{curl}_{\Gamma} \widehat{r}_{l}\right\|_{L_{2}\left(\Gamma^{+}\right)}^{2} .
$$

Now we have the means to switch from the splitting (6.4) to the modified decomposition

$$
\widetilde{\mathbf{v}}_{L}=\sum_{l=0}^{L} \widehat{\mathbf{w}}_{l}+\operatorname{curl}_{\Gamma} \widehat{q}_{l}, \quad \widehat{\mathbf{w}}_{l} \in \mathcal{R} \mathcal{T}_{0}\left(\Gamma_{l}\right), \widehat{q}_{l} \in \mathcal{S}_{1}\left(\Gamma_{l}\right),
$$

with

$$
\widehat{\mathbf{w}}_{l}:=\mathbf{w}_{l}-\operatorname{curl}_{\Gamma} \widehat{r}_{l} \quad, \quad \widehat{q}_{l}=q_{l}+\widehat{r}_{l} .
$$


Thanks to 6.10 it is clear that

$$
\left.\left(\sum_{l=0}^{L} \widehat{\mathbf{w}_{l}} \cdot \boldsymbol{n}_{\partial \Gamma^{+}}\right)\right|_{\partial \Gamma^{+}}=0 \quad,\left.\quad\left(\sum_{l=0}^{L} \widehat{q}_{l}\right)\right|_{\partial \Gamma^{+}}=0 .
$$

Now we appeal to the orthogonality property (6.1) for (6.10) in order to verify that the modification does not destroy the stability of the multilevel decompositions as follows:

$$
\begin{aligned}
& \sum_{l=0}^{L} h_{l}^{-1}\left\|\operatorname{curl}_{\Gamma} \widehat{r}_{l}\right\|_{L_{2}\left(\Gamma^{+}\right)}^{2} \\
& \stackrel{\sqrt{6.12)}}{\lesssim} \sum_{l=0}^{L}\left\|\operatorname{grad}_{\partial \Gamma^{+}} \check{r}_{l}\right\|_{L_{2}\left(\partial \Gamma^{+}\right)}^{2} \stackrel{\sqrt{6.17}}{=}\left\|\operatorname{grad}_{\partial \Gamma^{+}} \check{s}_{L}\right\|_{L_{2}\left(\partial \Gamma^{+}\right)}^{2} \\
& \stackrel{(6.16)}{=}\left\|\sum_{l=0}^{L} \mathbf{w}_{l} \cdot \boldsymbol{n}_{\partial \Gamma^{+}}\right\|_{L_{2}\left(\partial \Gamma^{+}\right)}^{2} \lesssim\left(\sum_{l=0}^{L}\left\|\mathbf{w}_{l} \cdot \boldsymbol{n}_{\partial \Gamma^{+}}\right\|_{L_{2}\left(\partial \Gamma^{+}\right)}\right)^{2} \\
& \lesssim \quad L \sum_{l=0}^{L}\left\|\mathbf{w}_{l} \cdot \boldsymbol{n}_{\partial \Gamma^{+}}\right\|_{L_{2}\left(\partial \Gamma^{+}\right)}^{2} \lesssim L \sum_{l=0}^{L} h_{l}^{-1}\left\|\mathbf{w}_{l}\right\|_{L_{2}\left(\Gamma^{+}\right)}^{2},
\end{aligned}
$$

where we used only scaling arguments and the Cauchy-Schwarz inequality in $\mathbb{R}^{L+1}$. Combined with (6.14) this permits us to conclude the stability estimate

$$
\sum_{l=0}^{L} h_{l}^{-1}\left(\left\|\widehat{\mathbf{w}}_{l}\right\|_{L_{2}\left(\Gamma^{+}\right)}^{2}+\left\|\widehat{q}_{l}\right\|_{L_{2}\left(\Gamma^{+}\right)}^{2}\right) \lesssim L \sum_{l=0}^{L} h_{l}^{-1}\left(\left\|\mathbf{w}_{l}\right\|_{L_{2}\left(\Gamma^{+}\right)}^{2}+\left\|q_{l}\right\|_{L_{2}\left(\Gamma^{+}\right)}^{2}\right) .
$$

3 Now we return to the reasoning of the proof of Theorem 3.6. in analogy to (3.15) we define $\widehat{\mathbf{u}}_{l} \in \mathcal{R} \mathcal{T}_{0}\left(\Gamma_{l}^{+}\right)$and $\widehat{p}_{l} \in \mathcal{S}_{1}\left(\Gamma_{l}^{+}\right)$by

$$
\widehat{\boldsymbol{u}}_{l}:=\sum_{k=0}^{l} \widehat{\mathbf{w}}_{l} \quad, \quad \widehat{p}_{l}:=\sum_{k=0}^{l} \widehat{q}_{l}, \quad l=0,1,2, \ldots, L .
$$

Next, taking the cue from (3.17) we introduce $\widetilde{\mathbf{w}}_{l} \in \widetilde{\mathcal{R}}_{0}\left(\Gamma_{l}^{+}\right), \widetilde{q}_{l} \in \widetilde{\mathcal{S}}_{1}\left(\Gamma_{l}^{+}\right), l=$ $0, \ldots, L$, according to

$$
\begin{gathered}
\widetilde{\mathbf{w}}_{l}:= \begin{cases}\mathrm{Z}_{0}^{1} \widehat{\boldsymbol{u}}_{0}, & \text { for } l=0, \\
\mathrm{Z}_{l}^{1} \widehat{\boldsymbol{u}}_{l}-\mathrm{Z}_{l-1}^{1} \widehat{\boldsymbol{u}}_{l-1}, & \text { for } l>0,\end{cases} \\
\widetilde{q}_{l}:= \begin{cases}\mathrm{Z}_{0}^{0} \widehat{q}_{0}, & \text { for } l=0, \\
\mathrm{Z}_{l}^{0} \widehat{q}_{l}-\mathrm{Z}_{l-1}^{0} \widehat{q}_{l-1}, & \text { for } l>0 .\end{cases}
\end{gathered}
$$

Recall the reasoning and manipulations underlying (3.18) and (3.19). In exactly the same way, we can show that

$$
\begin{aligned}
\sum_{l=0}^{L} h_{l}^{-1}\left\|\widetilde{\mathbf{w}}_{l}\right\|_{L_{2}(\Gamma)}^{2} & \lesssim L^{2} \sum_{l=0}^{L} h_{l}^{-1}\left\|\widehat{\mathbf{w}}_{l}\right\|_{L_{2}(\Gamma)}^{2} \\
& \lesssim L^{3} \sum_{l=0}^{L} h_{l}^{-1}\left(\left\|\mathbf{w}_{l}\right\|_{L_{2}\left(\Gamma^{+}\right)}^{2}+\left\|q_{l}\right\|_{L_{2}\left(\Gamma^{+}\right)}^{2}\right)
\end{aligned}
$$


with

$$
\sum_{l=0}^{L} \widetilde{\mathbf{w}}_{l}=\mathrm{Z}_{L}^{1} \widehat{\mathbf{u}}_{L}=\mathrm{Z}_{L}^{1}\left(\sum_{l=0}^{L} \widehat{\mathbf{w}}_{l}\right)=\sum_{l=0}^{L} \widehat{\mathbf{w}}_{l} .
$$

Here, owing to (6.16), we could drop the cut-off operator. This apparently insignificant step forced us to include part 2 of the proof.

The same techniques can be used to obtain the required estimate for the $\widehat{q}_{l}$ 's:

$$
\begin{aligned}
\sum_{l=0}^{L} h_{l}^{-1}\left\|\widetilde{q}_{l}\right\|_{L_{2}(\Gamma)}^{2} & \lesssim L^{2} \sum_{l=0}^{L} h_{l}^{-1}\left\|\check{q}_{l}\right\|_{L_{2}(\Gamma)}^{2} \\
& \lesssim L^{3} \sum_{l=0}^{L} h_{l}^{-1}\left(\left\|\mathbf{w}_{l}\right\|_{L_{2}\left(\Gamma^{+}\right)}^{2}+\left\|q_{l}\right\|_{L_{2}\left(\Gamma^{+}\right)}^{2}\right) .
\end{aligned}
$$

with

$$
\sum_{l=0}^{L} \widetilde{q}_{l}=\mathrm{Z}_{L}^{0}\left(\sum_{l=0}^{L} \widehat{q}_{l}\right) \stackrel{(6.16)}{=} \sum_{l=0}^{L} \widehat{q}_{l} .
$$

From (6.15), (6.20), and (6.21) we see that $\widetilde{\mathbf{w}}_{l} \in \widetilde{\mathcal{R T}}_{0}\left(\Gamma_{l}^{+}\right)$and $\widetilde{q}_{l} \in \widetilde{\mathcal{S}}_{1}\left(\Gamma_{l}^{+}\right)$really provide a valid multilevel decomposition of $\widetilde{\mathbf{v}}_{L}$ in the sense of (6.5). Then (6.3) results from combining (6.22) and (6.23).

Remark 6.1. What is the obstacle to obtain a cut-off estimate in Theorem 2.1? It is the fact that the cut-offs $Z_{l}^{0}, Z_{l}^{1}$ and $\operatorname{curl}_{\Gamma}$ do not commute. Hence, applying $Z_{L}^{0}$ to $\sum_{l=0}^{L} q_{l}$ in Step 2 of the proof would also affect edge associated degrees of freedom in the interior of $\Gamma^{+}$. This difficulty is connected with the need to rely on discrete scalar potential functions $\left(q_{l} / \widetilde{q}_{l}\right)$ in the multilevel characterizations of the norms $\|\cdot\|_{\boldsymbol{H}^{-\frac{1}{2}}\left(\operatorname{div}_{\Gamma}, \Gamma\right)}$ and $\|\cdot\|_{\boldsymbol{H}^{-\frac{1}{2}}\left(\operatorname{div}_{\Gamma}, \Gamma^{+}\right)}$on $\mathcal{R} \mathcal{T}_{0}\left(\Gamma_{L}\right)$ and $\mathcal{R} \mathcal{T}_{0}\left(\Gamma_{L}^{+}\right)$, respectively; cf. Theorems 5.1 and 5.2 .

This obstruction to extending Theorem 3.6 to $\mathcal{R} \mathcal{T}_{0}\left(\Gamma_{L}^{+}\right)$also enforces the use of so-called "barbed wire baskets" in sub-structuring domain decomposition methods for edge elements [17, 18, 32, 33.

Proof of Theorem 2.2. Of course, we follow the ideas in the proofs of Theorems 3.6 and 2.1. Appealing to Theorems 5.6 and 5.5, and analogous to (3.11) and (6.3), we have to prove that

$$
\left\|\widetilde{\varphi}_{L}\right\|_{\widetilde{\mathcal{Q}}_{0}\left(\Gamma_{L}^{+}\right)}^{2} \lesssim L^{3}\left\|\widetilde{\varphi}_{L}\right\|_{\mathcal{Q}_{0}\left(\Gamma_{L}^{+}\right)}^{2} \quad \forall \widetilde{\varphi}_{L} \in \widetilde{\mathcal{Q}}_{0}\left(\Gamma_{L}^{+}\right) .
$$

Given $\widetilde{\varphi}_{L} \in \widetilde{\mathcal{Q}}_{0}\left(\Gamma_{L}^{+}\right)$consider a multilevel decomposition

$$
\left.\widetilde{\varphi}_{L}\right|_{\Gamma^{+}}=\left.\left(\sum_{l=0}^{L}\left(\eta_{l}+\operatorname{div}_{\Gamma} \mathbf{q}_{l}\right)\right)\right|_{\Gamma^{+}}, \eta_{l} \in \mathcal{Q}_{0}\left(\Gamma_{l}\right), \mathbf{q}_{l} \in \mathcal{R} \mathcal{T}_{0}\left(\Gamma_{l}\right),
$$

of the form relevant for the definition of $\left\|\mid \widetilde{\varphi}_{L}\right\|_{\mathcal{Q}_{0}\left(\Gamma_{L}^{+}\right)}$in (5.20).

Parallel to step 2 of the proof of Theorem 2.1 we note that, in general,

$$
\left.\left(\mathbf{w}_{L} \cdot \boldsymbol{n}_{\partial \Gamma^{+}}\right)\right|_{\partial \Gamma^{+}} \neq 0 \quad \text { for } \quad \mathbf{w}_{L}:=\sum_{l=0}^{L} \mathbf{q}_{l} ;
$$


cf. 6.8). Again, we aim to modify the $\mathbf{q}_{l}$ 's in order to enforce the vanishing normal component on $\partial \Gamma^{+}$for their sum. To begin with, we find $\mathbf{c}_{0} \in \mathcal{R} \mathcal{T}_{0}\left(\Gamma_{0}^{+}\right)$(on the coarsest level!) such that

$$
\int_{\partial \Gamma^{+}} \mathbf{w}_{L} \cdot \boldsymbol{n}_{\partial \Gamma^{+}} \mathrm{d} l=\int_{\partial \Gamma^{+}} \mathbf{c}_{0} \cdot \boldsymbol{n}_{\partial \Gamma^{+}} \mathrm{d} l
$$

and, abusing notation, replace $\eta_{0} \leftarrow \eta_{0}+\operatorname{div}_{\Gamma} \mathbf{c}_{0}, \mathbf{q}_{0} \leftarrow \mathbf{q}_{0}-\mathbf{c}_{0}$. Hence for this new decomposition (6.25) still holds, and, since the modifications have been confined to level 0 , the change of the $h_{l}^{-1}$ weighted sum of the squared $L^{2}$-norms occurring in (5.16) and (5.20) remains under control. Reusing the definition of $\mathbf{w}_{L}$ from (6.26) we now know that

$$
\int_{\partial \Gamma^{+}} \mathbf{w}_{L} \cdot \boldsymbol{n}_{\partial \Gamma^{+}} \mathrm{d} l=0
$$

which ensures the existence of $\check{s}_{L} \in \mathcal{S}_{1}\left(\partial \Gamma_{L}^{+}\right)$such that

$$
\operatorname{grad}_{\partial \Gamma^{+}} \check{s}_{L}=\left.\left(\mathbf{w}_{L} \cdot \boldsymbol{n}_{\partial \Gamma^{+}}\right)\right|_{\partial \Gamma^{+}} .
$$

Next, we use the hierarchical decomposition of $\check{s}_{L}$ as introduced in the beginning of this section, and invoke (6.1)

$$
\check{s}_{L}=\sum_{l=0}^{L} \check{r}_{l}, \quad \sum_{l=0}^{L}\left\|\operatorname{grad}_{\partial \Gamma^{+}} \check{r}_{l}\right\|_{L_{2}\left(\partial \Gamma^{+}\right)}^{2}=\left\|\operatorname{grad}_{\partial \Gamma^{+}} \check{s}_{L}\right\|_{L_{2}\left(\partial \Gamma^{+}\right)}^{2} .
$$

We denote by $\widehat{r}_{l} \in \mathcal{S}_{1}\left(\Gamma_{l}^{+}\right)$the trivial nodal extension of $\check{r}_{l}$ according to (6.11) and recall the estimate (6.12). Using this, we can proceed as in (6.17) and end up with

$$
\sum_{l=0}^{L} h_{l}^{-1}\left\|\operatorname{curl}_{\Gamma} \widehat{r}_{l}\right\|_{L_{2}\left(\Gamma^{+}\right)}^{2} \lesssim L \sum_{l=0}^{L} h_{l}^{-1}\left\|\mathbf{q}_{l}\right\|_{L_{2}\left(\Gamma^{+}\right)}^{2} .
$$

Now set $\widehat{\mathbf{q}}_{l}:=\mathbf{q}_{l}-\operatorname{curl}_{\Gamma} \widehat{r}_{l} \in \mathcal{R} \mathcal{T}_{0}\left(\Gamma_{l}\right)$, which, as $\operatorname{div}_{\Gamma} \operatorname{curl}_{\Gamma} \widehat{r}_{l}=0$, yields a modified decomposition

$$
\left.\widetilde{\varphi}_{L}\right|_{\Gamma^{+}}=\left.\left(\sum_{l=0}^{L}\left(\eta_{l}+\operatorname{div}_{\Gamma} \widehat{\mathbf{q}}_{l}\right)\right)\right|_{\Gamma^{+}},
$$

which, by (6.30), still enjoys the stability

$$
\sum_{l=0}^{L} h_{l}^{-1}\left\|\widehat{\mathbf{q}}_{l}\right\|_{L_{2}\left(\Gamma^{+}\right)}^{2} \lesssim L \sum_{l=0}^{L} h_{l}^{-1}\left\|\mathbf{q}_{l}\right\|_{L_{2}\left(\Gamma^{+}\right)}^{2}
$$

and for which

$$
\left.\left(\sum_{l=0}^{L} \widehat{\mathbf{q}}_{l} \cdot \boldsymbol{n}_{\partial \Gamma^{+}}\right)\right|_{\partial \Gamma^{+}}=0 .
$$

Now we just follow Part 3 of the proof of Theorem 2.1, as in (6.20) and (6.21) modify the $\widehat{\mathbf{q}}_{l}$ in order to enforce zero normal components on $\partial \Gamma^{+}$, pay with another factor of $L^{2}$ in the estimate, as in (6.22), and we are done. 


\section{REFERENCES}

[1] Mark Ainsworth, Johnny Guzmán, and Francisco-Javier Sayas, Discrete extension operators for mixed finite element spaces on locally refined meshes, arXiv:1406.5534v2 [math.NA] (2015).

[2] M. Ainsworth and W. McLean, Multilevel diagonal scaling preconditioners for boundary element equations on locally refined meshes, Numer. Math. 93 (2003), no. 3, 387-413, DOI 10.1007/s002110100391. MR 1953746 (2004i:65130)

[3] A. Alonso and A. Valli, An optimal domain decomposition preconditioner for low-frequency time-harmonic Maxwell equations, Math. Comp. 68 (1999), no. 226, 607-631, DOI 10.1090/S0025-5718-99-01013-3. MR 1609607 (99i:78002)

[4] D. N. Arnold, R. S. Falk, and R. Winther, Multigrid in H(div) and H(curl), Numer. Math. 85 (2000), no. 2, 197-217, DOI 10.1007/PL00005386. MR.1754719 (2001d:65161)

[5] D. N. Arnold, R. S. Falk, and R. Winther, Multigrid preconditioning in H(div) on non-convex polygons, Comput. Appl. Math. 17 (1998), no. 3, 303-315. MR 1687885 (2000k:65224)

[6] J. Bey, Tetrahedral grid refinement (English, with English and German summaries), Computing 55 (1995), no. 4, 355-378, DOI 10.1007/BF02238487. MR1370107 (96i:65105)

[7] F. Bornemann and H. Yserentant, A basic norm equivalence for the theory of multilevel methods, Numer. Math. 64 (1993), no. 4, 455-476, DOI 10.1007/BF01388699. MR1213412 (94b:65155)

[8] J. H. Bramble, Multigrid Methods, Pitman Research Notes in Mathematics Series, vol. 294, Longman Scientific \& Technical, Harlow; copublished in the United States with John Wiley \& Sons, Inc., New York, 1993. MR1247694(95b:65002)

[9] J. H. Bramble, J. E. Pasciak, and A. H. Schatz, The construction of preconditioners for elliptic problems by substructuring. IV, Math. Comp. 53 (1989), no. 187, 1-24, DOI 10.2307/2008346. MR.970699 (89m:65098)

[10] F. Brezzi and M. Fortin, Mixed and Hybrid Finite Element Methods, Springer Series in Computational Mathematics, vol. 15, Springer-Verlag, New York, 1991. MR1115205 (92d:65187)

[11] A. Buffa and P. Ciarlet Jr., On traces for functional spaces related to Maxwell's equations. I. An integration by parts formula in Lipschitz polyhedra, Math. Methods Appl. Sci. 24 (2001), no. 1, 9-30, DOI 10.1002/1099-1476(20010110)24:1〈9::AID-MMA191〉3.0.CO;2-2. MR.1809491(2002b:78024)

[12] A. Buffa and P. Ciarlet Jr., On traces for functional spaces related to Maxwell's equations. II. Hodge decompositions on the boundary of Lipschitz polyhedra and applications, Math. Methods Appl. Sci. 24 (2001), no. 1, 31-48, DOI 10.1002/1099-1476(20010110)24:1;9::AIDMMA191¿3.0.CO;2-2. MR:1809492 (2002b:78025)

[13] A. Buffa, M. Costabel, and D. Sheen, On traces for $\mathbf{H}(\mathbf{c u r l}, \Omega)$ in Lipschitz domains, J. Math. Anal. Appl. 276 (2002), no. 2, 845-867, DOI 10.1016/S0022-247X(02)00455-9. MR.1944792 (2004i:35045)

[14] H. Chen, R. H. W. Hoppe, and X. Xu, Uniform convergence of local multigrid methods for the time-harmonic Maxwell equation, ESAIM Math. Model. Numer. Anal. 47 (2013), no. 1, 125-147, DOI 10.1051/m2an/2012023. MR2968698

[15] Choi-Hong Lai, Petter E. Bjørstad, Mark Cross, and Olof Widlund (eds.), Eleventh International Conference on Domain Decomposition Methods, DDM.org, Augsburg, 1999. Available electronically at http://www.ddm.org/DD11/index.html. MR.1827403

[16] S. H. Christiansen and R. Winther, Smoothed projections in finite element exterior calculus, Math. Comp. 77 (2008), no. 262, 813-829, DOI 10.1090/S0025-5718-07-02081-9. MR2373181 (2009a:65310)

[17] C. R. Dohrmann and O. B. Widlund, An iterative substructuring algorithm for twodimensional problems in H(curl), SIAM J. Numer. Anal. 50 (2012), no. 3, 1004-1028, DOI 10.1137/100818145. MR2970732

[18] C. Dohrmann and O. Widlund, Some recent tools and a BDDC algorithm for 3D problems in $\mathbb{H}($ curl), in Domain Decomposition Methods in Science and Engineering XX, R. Bank, M. Holst, O. Widlund, and J. Xu, eds., vol. 91 of Lecture Notes in Computational Science and Engineering, Springer, Berlin, Heidelberg, 2013, pp. 15-25. 
[19] M. Dryja, A method of domain decomposition for three-dimensional finite element elliptic problems, First International Symposium on Domain Decomposition Methods for Partial Differential Equations (Paris, 1987), SIAM, Philadelphia, PA, 1988, pp. 43-61. MR.972511 (90b:65200)

[20] A. Ern and J.-L. Guermond, Theory and Practice of Finite Elements, Applied Mathematical Sciences, vol. 159, Springer-Verlag, New York, 2004. MR2050138 (2005d:65002)

[21] V. Girault and P.-A. Raviart, Finite Element Methods for Navier-Stokes Equations, Theory and Algorithms, Springer Series in Computational Mathematics, vol. 5, Springer-Verlag, Berlin, 1986. MR851383 (88b:65129)

[22] P. Grisvard, Singularities in Boundary Value Problems, Recherches en Mathématiques Appliquées [Research in Applied Mathematics], vol. 22, Masson, Paris; Springer-Verlag, Berlin, 1992. MR:1173209 (93h:35004)

[23] N. Heuer, E. P. Stephan, and T. Tran, Multilevel additive Schwarz method for the h-p version of the Galerkin boundary element method, Math. Comp. 67 (1998), no. 222, 501-518, DOI 10.1090/S0025-5718-98-00926-0. MR.1451325 (98g:65108)

[24] R. Hiptmair, Multigrid method for $\mathbf{H}$ (div) in three dimensions, Electron. Trans. Numer. Anal. 6 (1997), no. Dec., 133-152. Special issue on multilevel methods (Copper Mountain, CO, 1997). MR 1615161 (99c:65232)

[25] R. Hiptmair, Multigrid method for Maxwell's equations, SIAM J. Numer. Anal. 36 (1999), no. 1, 204-225, DOI 10.1137/S0036142997326203. MR1654571 (99j:65229)

[26] R. Hiptmair, Finite elements in computational electromagnetism, Acta Numer. 11 (2002), 237-339, DOI 10.1017/S0962492902000041. MR2009375(2004k:78028)

[27] R. Hiptmair, Analysis of multilevel methods for eddy current problems, Math. Comp. 72 (2003), no. 243, 1281-1303, DOI 10.1090/S0025-5718-02-01468-0. MR.1972736 (2004c:78033)

[28] R. Hiptmair and C. Jerez-Hanckes, Multiple traces boundary integral formulation for Helmholtz transmission problems, Adv. Comput. Math. 37 (2012), no. 1, 39-91, DOI 10.1007/s10444-011-9194-3. MR2927645

[29] R. Hiptmair and S. Mao, Stable multilevel splittings of boundary edge element spaces, BIT 52 (2012), no. 3, 661-685, DOI 10.1007/s10543-012-0369-1. MR2965296

[30] R. Hiptmair and W.-Y. Zheng, Local multigrid in H(curl), Tech. Rep. 2007-03, SAM, ETH Zürich, Switzerland, March 2007. http://arxiv.org/abs/0901.0764.

[31] R. Hiptmair and W. Zheng, Local multigrid in $\mathbf{H}(\mathbf{c u r l})$, J. Comput. Math. 27 (2009), no. 5, 573-603, DOI 10.4208/jcm.2009.27.5.012. MR2536903(2010h:65249)

[32] Q. Hu and J. Zou, A non-overlapping domain decomposition method for Maxwell's equation in three dimensions, SIAM J. Numer. Anal., 41 (2003), pp. 1682-1708.

[33] Q. Hu and J. Zou, Substructuring preconditioners for saddle-point problems arising from Maxwell's equations in three dimensions, Math. Comp. 73 (2004), no. 245, 35-61, DOI 10.1090/S0025-5718-03-01541-2. MR2034110 (2004m:65197)

[34] I. Kossaczký, A recursive approach to local mesh refinement in two and three dimensions, J. Comput. Appl. Math. 55 (1994), no. 3, 275-288, DOI 10.1016/0377-0427(94)90034-5. MR.1329875 (95m:65207)

[35] J. Lions and F. Magenes, Nonhomogeneous boundary value problems and applications, Springer-Verlag, Berlin, 1972.

[36] W. McLean, Strongly elliptic systems and boundary integral equations, Cambridge University Press, Cambridge, 2000. MR 1742312(2001a:35051)

[37] W. McLean and O. Steinbach, Boundary element preconditioners for a hypersingular integral equation on an interval, Adv. Comput. Math. 11 (1999), no. 4, 271-286, DOI 10.1023/A:1018944530343. MR:1732138(2000k:65236)

[38] J.-C. Nédélec, Mixed finite elements in $\mathbf{R}^{3}$, Numer. Math. 35 (1980), no. 3, 315-341, DOI 10.1007/BF01396415. MR592160 (81k:65125)

[39] P. Oswald, On function spaces related to finite element approximation theory (English, with German and Russian summaries), Z. Anal. Anwendungen 9 (1990), no. 1, 43-64. MR1063242 (91g:65246)

[40] P. Oswald, On discrete norm estimates related to multilevel preconditioners in the finite element method, in Constructive Theory of Functions, Proc. Int. Conf. Varna 1991, K. Ivanov, P. Petrushev, and B. Sendov, eds., Bulg. Acad. Sci., 1992, pp. 203-214. 
[41] P. Oswald, Multilevel finite element approximation, Teubner Skripten zur Numerik. [Teubner Scripts on Numerical Mathematics], B. G. Teubner, Stuttgart, 1994. Theory and applications. MR.1312165 (95k:65110)

[42] S. A. Sauter and C. Schwab, Boundary Element Methods, Springer Series in Computational Mathematics, vol. 39, Springer-Verlag, Berlin, 2011. Translated and expanded from the 2004 German original. MR2743235 (2011i:65003)

[43] L. R. Scott and S. Zhang, Finite element interpolation of nonsmooth functions satisfying boundary conditions, Math. Comp. 54 (1990), no. 190, 483-493, DOI 10.2307/2008497. MR.1011446 (90j:65021)

[44] L. Tartar, An Introduction to Sobolev Spaces and Interpolation Spaces, Lecture Notes of the Unione Matematica Italiana, vol. 3, Springer, Berlin; UMI, Bologna, 2007. MR2328004 (2008g:46055)

[45] A. Toselli and O. Widlund, Domain Decomposition Methods-Algorithms and Theory, Springer Series in Computational Mathematics, vol. 34, Springer-Verlag, Berlin, 2005. MR2104179 (2005g:65006)

[46] J. Xu, Iterative methods by space decomposition and subspace correction, SIAM Rev. 34 (1992), no. 4, 581-613, DOI 10.1137/1034116. MR.1193013 (93k:65029)

[47] J. Xu and J. Zou, Some nonoverlapping domain decomposition methods, SIAM Rev. 40 (1998), no. 4, 857-914, DOI 10.1137/S0036144596306800. MR:1659681 (99m:65241)

[48] J. Xu, L. Chen, and R. H. Nochetto, Optimal multilevel methods for H(grad), H(curl), and $H($ div) systems on graded and unstructured grids, Multiscale, nonlinear and adaptive approximation, Springer, Berlin, 2009, pp. 599-659, DOI 10.1007/978-3-642-03413-8_14. MR2648382 (2011k:65178)

[49] X. Zhang, Multilevel Schwarz methods, Numer. Math. 63 (1992), no. 4, 521-539, DOI 10.1007/BF01385873. MR1189535 (93h:65047)

SAM, ETH ZÜRICH, CH-8092 ZüRICH, SwITZERLAND

E-mail address: hiptmair@sam.math.ethz.ch

School of Engineering, Pontificia Universidad Católica de Chile, Santiago, Chile

E-mail address: cjerez@ing.puc.cl

LSEC, Institute of Computational Mathematics, Academy of Mathematics and System Science, Chinese Academy of Sciences, Beijing, 100190, People's Republic of China

E-mail address: maosp@lses.cc.ac.cn 